\title{
REPRESENTATION ZETA FUNCTIONS OF SOME COMPACT $p$-ADIC ANALYTIC GROUPS
}

\author{
NIR AVNI, BENJAMIN KLOPSCH, URI ONN, AND CHRISTOPHER VOLL
}

\begin{abstract}
Using the Kirillov orbit method, novel methods from p-adic integration and Clifford theory, we study representation zeta functions associated to compact $p$-adic analytic groups. In particular, we give general estimates for the abscissae of convergence of such zeta functions.

We compute explicit formulae for the representation zeta functions of some compact $p$-adic analytic groups, defined over a compact discrete valuation ring $\mathfrak{o}$ of characteristic 0 . These include principal congruence subgroups of $\mathrm{SL}_{2}(\mathfrak{o})$, without any restrictions on the residue field characteristic of $\mathfrak{o}$, as well as the norm one group $\mathrm{SL}_{1}(\mathfrak{D})$ of a nonsplit quaternion algebra $\mathfrak{D}$ over the field of fractions of $\mathfrak{o}$ and its principal congruence subgroups. We also determine the representation zeta functions of principal congruence subgroups of $\mathrm{SL}_{3}(\mathfrak{o})$ in the case that $\mathfrak{o}$ has residue field characteristic 3 and is unramified over $\mathbb{Z}_{3}$.
\end{abstract}

\section{INTRODUCTION}

Let $G$ be a group. For $n \in \mathbb{N}$, we denote by $r_{n}(G)$ the number of isomorphism classes of $n$-dimensional irreducible complex representations of $G$. If $G$ is a topological group, we tacitly assume that representations are continuous. We call $G$ (representation) rigid if, for every $n \in \mathbb{N}$, the number $r_{n}(G)$ is finite. It is known that a profinite group is rigid if and only if it is FAb, i.e. if each of its open subgroups has finite abelianisation. All the groups studied in this paper are rigid.

We say that $G$ has polynomial representation growth if the sequence $R_{N}(G):=$ $\sum_{n=1}^{N} r_{n}(G)$ is bounded by a polynomial in $N$. The representation zeta function of a group $G$ with polynomial representation growth is the Dirichlet series

$$
\zeta_{G}(s):=\sum_{n=1}^{\infty} r_{n}(G) n^{-s},
$$

where $s$ is a complex variable. The abscissa of convergence $\alpha(G)$ of $\zeta_{G}(s)$, i.e. the infimum of all $\alpha \in \mathbb{R}$ such that $\zeta_{G}(s)$ converges on the complex right half-plane $\{s \in \mathbb{C} \mid$ $\operatorname{Re}(s)>\alpha$, gives the precise degree of polynomial growth: $\alpha(G)$ is the smallest $\alpha$ such that $R_{n}(G)=O\left(1+n^{\alpha+\varepsilon}\right)$ for every $\varepsilon \in \mathbb{R}_{>0}$. Representation zeta functions of groups have been studied in a variety of contexts, e.g. in the setting of arithmetic and $p$-adic analytic groups, wreath products of finite groups and finitely generated nilpotent groups; cf. $[12,16,2,3,4,[5]$ and [9, 19]. In the case of finitely generated nilpotent groups, one enumerates representations up to twisting by one-dimensional representations.

2000 Mathematics Subject Classification. 22E50, 20F69, 11M41, 20C15, $20 \mathrm{G} 25$.

Key words and phrases. Representation growth, p-adic analytic group, Igusa local zeta function, $\mathfrak{p}$-adic integration, Kirillov orbit method.

Avni was supported by NSF grant DMS-0901638. 
We state our main results, deferring the precise definitions of some of the technical terms involved as well as further corollaries and remarks to later sections. In our first theorem we provide general estimates for the abscissae of convergence of representation zeta functions of FAb compact $p$-adic analytic groups. These are expressed in terms of invariants $\rho$ and $\sigma$ which are defined by minimal and maximal centraliser dimensions in the corresponding Lie algebras; see (2.4). The term 'permissible' is defined in Section 2.1; here it suffices to note that, for a given Lie lattice $\mathfrak{g}$, all sufficiently large $m \in \mathbb{N}$ are permissible for $\mathfrak{g}$.

Theorem 1.1. Let $\mathfrak{o}$ be a compact discrete valuation ring of characteristic 0 , with maximal ideal $\mathfrak{p}$ and field of fractions $\mathfrak{k}$. Let $\mathfrak{g}$ be an $\mathfrak{o}$-Lie lattice such that $\mathfrak{k} \otimes_{\mathfrak{o}} \mathfrak{g}$ is a perfect Lie algebra. Let $d:=\operatorname{dim}_{\mathfrak{k}}\left(\mathfrak{k} \otimes_{\mathfrak{o}} \mathfrak{g}\right)$, and let $\sigma:=\sigma(\mathfrak{g})$ and $\rho:=\rho(\mathfrak{g})$, as defined in (2.4). Let $m \in \mathbb{N}$ be permissible for $\mathfrak{g}$, and let $\mathrm{G}^{m}:=\exp \left(\mathfrak{p}^{m} \mathfrak{g}\right)$.

Then lower and upper bounds for the abscissa of convergence of $\zeta_{\mathrm{G}^{m}}(s)$ are given by

$$
(d-2 \rho) \rho^{-1} \leq \alpha\left(\mathrm{G}^{m}\right) \leq(d-2 \sigma) \sigma^{-1} .
$$

Our other main results provide explicit formulae for the representation zeta functions of members of specific families of 'simple' compact $p$-adic analytic groups. The groups are defined over a compact discrete valuation ring $\mathfrak{o}$ of characteristic 0 and residue field characteristic $p$, with absolute ramification index $e\left(\mathfrak{o}, \mathbb{Z}_{p}\right)$. In the unramified case $e\left(\mathfrak{o}, \mathbb{Z}_{p}\right)=1$ all $m \in \mathbb{N}$ are permissible for a given $\mathfrak{o}$-Lie lattice $\mathfrak{g}$.

Theorem 1.2. Let $\mathfrak{o}$ be a compact discrete valuation ring of characteristic 0 . Then for all $m \in \mathbb{N}$ which are permissible for the Lie lattice $\mathfrak{s l}_{2}(\mathfrak{o})$ the representation zeta function of the principal congruence subgroup $\mathrm{SL}_{2}^{m}(\mathfrak{o})$ is

$$
\zeta_{\mathrm{SL}_{2}^{m}(\mathfrak{o})}(s)= \begin{cases}q^{3 m} \frac{1-q^{-2-s}}{1-q^{1-s}} & \text { if } p>2, \\ q^{3 m \frac{q^{2}-q^{-s}}{1-q^{1-s}}} & \text { if } p=2 \text { and } e\left(\mathfrak{o}, \mathbb{Z}_{2}\right)=1 .\end{cases}
$$

Theorem 1.3. Let $\mathfrak{D}$ be a non-split quaternion algebra over a $\mathfrak{p}$-adic field $\mathfrak{k}$. Let $\mathfrak{R}$ denote the maximal compact subring of $\mathfrak{D}$, and suppose that $2 e\left(\mathfrak{k}, \mathbb{Q}_{p}\right)<p-1$, where $p$ denotes the residue field characteristic of $\mathfrak{k}$.

Then the representation zeta functions of $\mathrm{SL}_{1}(\mathfrak{D})=\mathrm{SL}_{1}(\mathfrak{R})$ and its principal congruence subgroups $\mathrm{SL}_{1}^{m}(\mathfrak{R}), m \in \mathbb{N}$, are

$$
\begin{aligned}
\zeta_{\mathrm{SL}_{1}(\mathfrak{R})}(s) & =\frac{(q+1)\left(1-q^{-s}\right)+4(q-1)((q+1) / 2)^{-s}}{1-q^{1-s}}, \\
\zeta_{\mathrm{SL}_{1}^{m}(\mathfrak{R})}(s) & =q^{3 m} \frac{q-q^{-1-s}}{1-q^{1-s}} .
\end{aligned}
$$

Theorem 1.4. Let $\mathfrak{o}$ be a compact discrete valuation ring of characteristic 0 , with residue field of cardinality $q$ and characteristic 3 . Suppose that $\mathfrak{o}$ is unramified over $\mathbb{Z}_{3}$. Then, for all $m \in \mathbb{N}$, one has

$$
\zeta_{\mathrm{SL}_{3}^{m}(\mathfrak{o})}(s)=q^{8 m-4} \frac{\left(q^{2}-q^{-s}\right)\left(q^{2}+q^{-s}+\left(q^{4}-1\right) q^{-2 s}-q^{1-3 s}+\left(q^{4}-q^{2}-q\right) q^{-4 s}\right)}{\left(1-q^{1-2 s}\right)\left(1-q^{2-3 s}\right)} .
$$

The four theorems are proved and discussed in Sections 2.3, 3.1, 4.2 and 5respectively. Whilst all of the results in the current paper are of a local nature, they might be best appreciated in the 'global' context of representation zeta functions of arithmetic groups, as explained to some extent below and exposed, in much greater detail, in [2, 3, 4]. 
The main technical tools of these papers are the Kirillov orbit method, novel techniques from $\mathfrak{p}$-adic integration and Clifford theory. The current paper is closely related to these works, complements them in parts and provides concrete examples of the general methods developed in these papers. We tried, however, to keep it reasonably selfcontained, and hope that it might help the reader appreciate these articles and their interconnections.

Let $\Gamma$ be an arithmetic subgroup of a connected, simply connected semisimple algebraic group $\mathbf{G}$ defined over a number field $k$, and assume that $\Gamma$ has the Congruence Subgroup Property. Relevant examples are groups of the form $\Gamma=\mathrm{SL}_{n}(\mathcal{O})$, where $\mathcal{O}$ is the ring of integers in a number field $k$, and $n \geq 3$. The Congruence Subgroup Property and Margulis super-rigidity imply that the 'global' representation zeta function $\zeta_{\Gamma}(s)$ of $\Gamma$ is an Euler product of 'local' representation zeta functions, indexed by places of $k$; see [16. Proposition 1.3]. For example, if $\Gamma=\mathrm{SL}_{n}(0)$ with $n \geq 3$, we have

$$
\zeta_{\mathrm{SL}_{n}(\odot)}(s)=\zeta_{\mathrm{SL}_{n}(\mathbb{C})}(s)^{|k: \mathbb{Q}|} \cdot \prod_{v} \zeta_{\mathrm{SL}_{n}\left(\Theta_{v}\right)}(s)
$$

where each archimedean factor $\zeta_{\mathrm{SL}_{n}(\mathbb{C})}(s)$ enumerates the finite-dimensional, irreducible rational representations of the algebraic group $\mathrm{SL}_{n}(\mathbb{C})$ and, for every non-archimedean place $v$ of $k$, we denote by $\mathcal{O}_{v}$ the completion of $\mathcal{O}$ at $v$, which is a finite extension of the $p$-adic integers $\mathbb{Z}_{p}$ if $v$ prolongs $p$. In general, the local factors indexed by nonarchimedean places are representation zeta functions of FAb compact $p$-adic analytic groups. In [4] we provide explicit, uniform formulae for the zeta functions of groups of the form $\mathrm{SL}_{3}(\mathfrak{o})$, in the case that $p>\max \{3, e\}$, where $e=e\left(\mathfrak{o}, \mathbb{Z}_{p}\right)$ denotes the absolute ramification index of $\mathfrak{o}$. In the Euler product (1.1) this condition is satisfied by all but finitely many of the rings $\mathfrak{o}=\mathcal{O}_{v}$. It is a natural, interesting question to describe the local factors at the finitely many 'exceptional' places $v$ of $k$, and in particular in the non-generic case $p=3$.

In [3] we develop general methods to describe representation zeta functions of certain 'globally defined' FAb compact $p$-adic analytic pro- $p$ groups. These include the principal congruence subgroups of compact $p$-adic analytic groups featuring in Euler products of representation zeta functions of 'semisimple' arithmetic groups, such as (1.1). In particular, we obtain there formulae for the zeta functions of principal congruence subgroups of the form $\mathrm{SL}_{3}^{m}(\mathfrak{o})$, provided the residue field characteristic of $\mathfrak{o}$ is different from 3 and $m \in \mathbb{N}$ is permissible for the $\mathfrak{o}$-Lie lattice $\mathfrak{s l}_{3}(\mathfrak{o})$, cf. [3, Theorem E].

In Theorem 1.4 of the current paper we complement [3, Theorem E] (or, equivalently, the analogous result in [4]) by providing a formula for the representation zeta functions of groups of the form $\mathrm{SL}_{3}^{m}(\mathfrak{o})$, where $\mathfrak{o}$ is an unramified extension of $\mathbb{Z}_{3}$ and $m \in \mathbb{N}$. The formula differs from the 'generic' formula in [3, Theorem E], valid for residue field characteristic $p \neq 3$, and is obtained by computations akin to the algebraic approach developed in [4]. It is noteworthy that the 'generic' formula only depends on the residue field of the local ring $\mathfrak{o}$, irrespective of ramification. Whilst it is clear that this does not hold in the case of residue field characteristic 3, we do not know how sensitive the zeta function is to ramification in this case. It is, for instance, an interesting open problem whether, for rings $\mathfrak{o}_{1}$ and $\mathfrak{o}_{2}$ of residue field characteristic 3 which have the same inertia degree and ramification index over $\mathbb{Z}_{3}$, the representation zeta functions of 
the groups $\mathrm{SL}_{3}\left(\mathfrak{o}_{1}\right)$ and $\mathrm{SL}_{3}\left(\mathfrak{o}_{2}\right)$ are the same, or at least those of $\mathrm{SL}_{3}^{m}\left(\mathfrak{o}_{1}\right)$ and $\mathrm{SL}_{3}^{m}\left(\mathfrak{o}_{2}\right)$ for permissible $m \in \mathbb{N}$.

A phenomenon of the latter kind is exhibited in Theorem 1.2, in which we record formulae for zeta functions of groups of the form $\mathrm{SL}_{2}^{m}(\mathfrak{o})$, where $m \in \mathbb{N}$ is permissible for the Lie lattice $\mathfrak{s l}_{2}(\mathfrak{o})$. The formula for $\zeta_{\mathrm{SL}_{2}^{m}(\mathfrak{o})}(s)$ in the generic case $p>2$ only depends on $m$ and on $q$, the residue field cardinality of $\mathfrak{o}$. The expression in the case $p=2$, on the other hand, is sensitive to ramification, albeit only to the absolute ramification index $e=e\left(\mathfrak{o}, \mathbb{Z}_{2}\right)$ of $\mathfrak{o}$. In Section 3.3 we employ Clifford theory to compute the representation zeta function $\zeta_{\mathrm{SL}_{2}(\mathfrak{o})}(s)$ in the case that $p \geq e-2$. This reproduces, in the given case, a formula first computed in [12, Theorem 7.5] for the zeta functions of groups of the form $\mathrm{SL}_{2}(R)$, where $R$ is an arbitrary complete discrete valuation ring with finite residue field of odd characteristic. We record it here as it illustrates our broader and conceptually different approach. We note that this formula, too, only depends on the residue field cardinality and not, for instance, on ramification. It is a challenge to establish analogous formulae in residue field characteristic 2. We record a formula for the representation zeta functions of $\mathrm{SL}_{2}\left(\mathbb{Z}_{2}\right)$, based on [17, and a conjectural formula for its first principal congruence subgroup.

Our methods also allow for explicit calculations for representation zeta functions of norm one groups $\mathrm{SL}_{1}(\mathfrak{D})$ of central division algebras $\mathfrak{D}$ of Schur index $\ell$, say, over $\mathfrak{p}$ adic fields $\mathfrak{k}$, and their principal congruence subgroups. Results obtained by Larsen and Lubotzky in [16] suggest that such 'anisotropic' groups may actually be more tractable than their 'isotropic' counterparts. In the case that $\ell$ is prime, we compute the abscissa of convergence of $\zeta_{\mathrm{SL}_{1}(\mathfrak{D})}(s)$ in terms of Lie-theoretic data associated to the Lie algebra $\mathfrak{s l}_{1}(\mathfrak{D})$; cf. Corollary 4.1. This result, which was first proved in [16] for general $\ell$, is an easy application of some general estimates for the abscissae of convergence of representation zeta functions of groups to which our methods are applicable; cf. Theorem 1.1. Of special interest is the case $\ell=2$, where $\mathfrak{D}$ is a non-split quaternion algebra over $\mathfrak{k}$ with maximal compact subring $\mathfrak{R}$, say. In this situation we give, in Theorem 1.3, formulae for the representation zeta functions of the groups $\mathrm{SL}_{1}(\mathfrak{D})=\mathrm{SL}_{1}(\mathfrak{R})$ and $\mathrm{SL}_{1}^{m}(\mathfrak{R}), m \in \mathbb{N}$, which hold if $p$ is large compared to the ramification index $e\left(\mathfrak{k}, \mathbb{Q}_{p}\right)$.

Organisation. The paper is organised as follows. In Section 2 we briefly recall the geometric method, developed in [3], to describe representation zeta functions of certain compact $p$-adic analytic pro- $p$ groups. We show how it yields lower and upper bounds for abscissae of convergence as in Theorem 1.1. In Section 3 we compute representation zeta functions associated to groups of the form $\mathrm{SL}_{2}(\mathfrak{o})$ and its principal congruence subgroups, thereby proving Theorem [1.2, Results on representation zeta functions of subgroups of norm one groups of central division algebras and, in particular, Theorem 1.3 are obtained in Section 4. The computations for principal subgroups of groups of the form $\mathrm{SL}_{2}(\mathfrak{o})$ in residue field characteristic $p=3$, resulting in Theorem 1.4, are carried out in Section 5 ,

Notation. Our notation is the same as the one used in [3]. Non-standard terms are briefly defined at their first occurrence in the text. Zeta function will always refer to representation zeta function. Throughout this paper, $\mathfrak{o}$ denotes a compact discrete valuation ring of characteristic 0 and residue field cardinality $q$, a power of a prime $p$. We write $F^{*}$ to denote the multiplicative group of a field $F$ and extend this notation as follows. For a non-trivial o-module $M$ we write $M^{*}:=M \backslash \mathfrak{p} M$ and set $\{0\}^{*}=\{0\}$. 


\section{ZETA FUNCTIONS AS p-ADIC INTEGRALS}

Let $\mathfrak{o}$ be a compact discrete valuation ring of characteristic 0 , with maximal ideal $\mathfrak{p}$. The residue field $\mathfrak{o} / \mathfrak{p}$ is a finite field of characteristic $p$ and cardinality $q$, say. Let $\mathfrak{k}$ be the field of fractions of $\mathfrak{o}$.

2.1. Integral formula. Let $\mathfrak{g}$ be an $\mathfrak{o}$-Lie lattice such that $\mathfrak{k} \otimes_{\mathfrak{o}} \mathfrak{g}$ is perfect. In accordance with [3, Section 2.1], we call $m \in \mathbb{N}_{0}$ permissible for $\mathfrak{g}$ if the principal congruence Lie sublattice $\mathfrak{g}^{m}=\mathfrak{p}^{m} \mathfrak{g}$ is potent and saturable. Almost all non-negative integers $m$ are permissible for $\mathfrak{g}$; see [3, Proposition 2.3]. A key property of a potent and saturable $\mathfrak{o}$-Lie lattice $\mathfrak{h}$ is that the Kirillov orbit method can be used to study the set $\operatorname{Irr}(H)$ of irreducible complex characters of the $p$-adic analytic pro- $p$ group $H=\exp (\mathfrak{h})$, which is associated to $\mathfrak{h}$ via the Hausdorff series; see [8].

Let $m \in \mathbb{N}_{0}$ be permissible for $\mathfrak{g}$ and consider $\mathrm{G}^{m}:=\exp \left(\mathfrak{g}^{m}\right)$. Then the orbit method provides a correspondence between the elements of $\operatorname{Irr}\left(\mathrm{G}^{m}\right)$ and the co-adjoint orbits of $\mathrm{G}^{m}$ on the Pontryagin dual $\operatorname{Irr}\left(\mathfrak{g}^{m}\right)=\operatorname{Hom}\left(\mathfrak{g}^{m}, \mathbb{C}^{*}\right)$ of the compact abelian group $\mathfrak{g}^{m}$. The radical of $\omega \in \operatorname{Irr}\left(\mathfrak{g}^{m}\right)$ is $\operatorname{Rad}(\omega):=\left\{x \in \mathfrak{g}^{m} \mid \forall y \in \mathfrak{g}^{m}: \omega\left([x, y]_{\text {Lie }}\right)=1\right\}$. The degree of the irreducible complex character represented by the co-adjoint orbit of $\omega$ is equal to $\left|\mathfrak{g}^{m}: \operatorname{Rad}(\omega)\right|^{1 / 2}$, and the size of the co-adjoint orbit of $\omega$ is equal to $\left|\mathfrak{g}^{m}: \operatorname{Rad}(\omega)\right|$. This shows that the zeta function of $\mathrm{G}^{m}$ satisfies

$$
\zeta_{\mathrm{G}^{m}}(s)=\sum_{\omega \in \operatorname{Irr}\left(\mathfrak{g}^{m}\right)}\left|\mathfrak{g}^{m}: \operatorname{Rad}(\omega)\right|^{-(s+2) / 2} .
$$

According to [3, Lemma 2.4], the Pontryagin dual of the $\mathfrak{o}$-Lie lattice $\mathfrak{g}^{m}$ admits a natural decomposition

$$
\operatorname{Irr}\left(\mathfrak{g}^{m}\right)=\bigcup_{n \in \mathbb{N}_{0}} \operatorname{Irr}_{n}\left(\mathfrak{g}^{m}\right), \quad \text { where } \operatorname{Irr}_{n}\left(\mathfrak{g}^{m}\right) \cong \operatorname{Hom}_{\mathfrak{o}}\left(\mathfrak{g}^{m}, \mathfrak{o} / \mathfrak{p}^{n}\right)^{*}
$$

Moreover, for each $n \in \mathbb{N}_{0}$ there is a natural projection of $\mathfrak{o}$-modules $\operatorname{Hom}_{\mathfrak{o}}\left(\mathfrak{g}^{m}, \mathfrak{o}\right) \rightarrow$ $\operatorname{Hom}_{\mathfrak{o}}\left(\mathfrak{g}^{m}, \mathfrak{o} / \mathfrak{p}^{n}\right)$, mapping $\operatorname{Hom}_{\mathfrak{o}}\left(\mathfrak{g}^{m}, \mathfrak{o}\right)^{*}$ onto $\operatorname{Hom}_{\mathfrak{o}}\left(\mathfrak{g}^{m}, \mathfrak{o} / \mathfrak{p}^{n}\right)^{*}$. We say that $\omega \in \operatorname{Irr}_{n}\left(\mathfrak{g}^{m}\right)$ has level $n$ and that $w \in \operatorname{Hom}_{\mathfrak{o}}\left(\mathfrak{g}^{m}, \mathfrak{o}\right)^{*}$ is a representative of $\omega$ if $w$ maps onto the appropriate element of $\operatorname{Hom}_{\mathfrak{o}}\left(\mathfrak{g}^{m}, \mathfrak{o} / \mathfrak{p}^{n}\right)^{*}$.

Let $\mathbf{b}:=\left(b_{1}, \ldots, b_{d}\right)$ be an $\mathfrak{o}$-basis for the $\mathfrak{o}$-Lie lattice $\mathfrak{g}$, where $d=\operatorname{dim}_{\mathfrak{k}}\left(\mathfrak{k} \otimes_{\mathfrak{o}} \mathfrak{g}\right)$. The structure constants $\lambda_{i j}^{h}$ of the $\mathfrak{o}$-Lie lattice $\mathfrak{g}$ with respect to $\mathbf{b}$ are encoded in the commutator matrix

$$
\mathcal{R}(\mathbf{Y}):=\mathcal{R}_{\mathfrak{g}, \mathbf{b}}(\mathbf{Y})=\left(\sum_{h=1}^{d} \lambda_{i j}^{h} Y_{h}\right)_{i j} \in \operatorname{Mat}_{d}(\mathfrak{o}[\mathbf{Y}]),
$$

whose entries are linear forms in independent variables $Y_{1}, \ldots, Y_{d}$. We write $W(\mathfrak{o}):=$ $\left(\mathfrak{o}^{d}\right)^{*} \cong \operatorname{Hom}_{\mathfrak{o}}\left(\mathfrak{g}^{m}, \mathfrak{o}\right)^{*}$ and set

$$
\sigma(\mathfrak{g}):=\min \left\{\frac{1}{2} \operatorname{rk}_{\mathfrak{k}} \mathcal{R}(\mathbf{y}) \mid \mathbf{y} \in W(\mathfrak{o})\right\} \quad \text { and } \quad \rho(\mathfrak{g}):=\max \left\{\frac{1}{2} \operatorname{rk}_{\mathfrak{k}} \mathcal{R}(\mathbf{y}) \mid \mathbf{y} \in W(\mathfrak{o})\right\} .
$$

Note that this definition is independent of the choice of basis for $\mathfrak{g}$, and that both $\sigma(\mathfrak{g})$ and $\rho(\mathfrak{g})$ are integers, because $\mathcal{R}(\mathbf{Y})$ is anti-symmetric.

The relevance of the commutator matrix in connection with (2.1) stems from [3, Lemma 3.3], which we record here as follows.

Lemma 2.1. Let $m$ be permissible for $\mathfrak{g}$, let $n \in \mathbb{N}$ and suppose that $\omega \in \operatorname{Irr}_{n}\left(\mathfrak{g}^{m}\right)$ is represented by $w \in \operatorname{Hom}_{\mathfrak{o}}\left(\mathfrak{g}^{m}, \mathfrak{o}\right)^{*}$. Let $\pi$ denote a uniformiser for $\mathfrak{o}$. 
Then for every $z \in \mathfrak{g}^{m}$ we have

$$
z \in \operatorname{Rad}(\omega) \quad \Longleftrightarrow \quad \underline{z} \cdot \mathcal{R}(\underline{w}) \equiv_{\mathfrak{p}^{n-m}} 0,
$$

where $\underline{z}$ and $\underline{w}$ denote the coordinate tuples of $z$ and $w$ with respect to the shifted $\mathfrak{o}$-basis $\pi^{m} \mathbf{b}$ for $\mathfrak{g}^{m}$ and its dual $\pi^{-m} \mathbf{b}^{\vee}$ for $\operatorname{Hom}_{\mathfrak{o}}\left(\mathfrak{g}^{m}, \mathfrak{o}\right)$.

By this lemma, the index $\left|\mathfrak{g}^{m}: \operatorname{Rad}(\omega)\right|$ can be expressed in terms of the elementary divisors of the matrix $\mathcal{R}(\underline{w})$ which in turn one computes from its minors. The zeta function of the group $\mathrm{G}^{m}$, associated to the principal congruence Lie sublattice $\mathfrak{g}^{m}$, can thus be regarded as a Poincaré series encoding the numbers of solutions of a certain system of equations modulo $\mathfrak{p}^{n}$ for all $n \in \mathbb{N}_{0}$. Such Poincaré series can be expressed as generalised Igusa zeta functions, which are certain types of $\mathfrak{p}$-adic integrals over the compact space $\mathfrak{p} \times W(\mathfrak{o})$; cf. [3] and [7, 11].

For $j \in\{1, \ldots, \rho(\mathfrak{g})\}$ and $\mathbf{y} \in W(\mathfrak{o})$ we define

$$
\begin{aligned}
F_{j}(\mathbf{Y}) & =\{f \mid f \text { a } 2 j \times 2 j \text { minor of } \mathcal{R}(\mathbf{Y})\}, \\
\|F(\mathbf{y})\|_{\mathfrak{p}} & =\max \left\{|f(\mathbf{y})|_{\mathfrak{p}} \mid f \in F\right\} .
\end{aligned}
$$

It is worth pointing out that the sets $F_{j}(\mathbf{Y})$ may be replaced by sets of polynomials defining the same polynomial ideals. Specifically, one could define $F_{j}(\mathbf{Y})$ to be the set of all principal $2 j \times 2 j$ minors; see [3, Remark 3.16]. It is the geometry of the varieties defined by the polynomials in $F_{j}(\mathbf{Y})$ which largely determines the zeta function of $\mathrm{G}^{m}$. Of particular interest are 'effective' resolutions of their singularities; cf. [3]. If $\mathfrak{k} \otimes_{\mathfrak{o}} \mathfrak{g}$ is a semisimple Lie algebra, then the varieties defined by the polynomials in $F_{j}(\mathbf{Y})$ admit a Lie-theoretic interpretation: they yield a stratification of the Lie algebra defined in terms of centraliser dimensions; cf. [3, Section 5]. We state the integral formula derived in [3, Section 3.2].

Proposition 2.2. Let $\mathfrak{g}$ be an $\mathfrak{o}$-Lie lattice such that $\mathfrak{k} \otimes_{\mathfrak{o}} \mathfrak{g}$ is a perfect $\mathfrak{k}$-Lie algebra of dimension d. Then for every $m \in \mathbb{N}_{0}$ which is permissible for $\mathfrak{g}$ one has

$$
\zeta_{\mathrm{G}^{m}}(s)=q^{d m}\left(1+\left(1-q^{-1}\right)^{-1} z_{\mathfrak{o}}(-s / 2-1, \rho(s+2)-d-1)\right),
$$

with $\rho=\rho(\mathfrak{g})$ and

$$
z_{\mathfrak{o}}(r, t)=\int_{(x, \mathbf{y}) \in \mathfrak{p} \times W(\mathfrak{o})}|x|_{\mathfrak{p}}^{t} \prod_{1 \leq j \leq \rho(\mathfrak{g})} \frac{\left\|F_{j}(\mathbf{y}) \cup F_{j-1}(\mathbf{y}) x^{2}\right\|_{\mathfrak{p}}^{r}}{\left\|F_{j-1}(\mathbf{y})\right\|_{\mathfrak{p}}^{r}} d \mu(x, \mathbf{y}),
$$

where $\mathfrak{p} \times W(\mathfrak{o}) \subseteq \mathfrak{o}^{d+1}$ and the additive Haar measure $\mu$ is normalised to $\mu\left(\mathfrak{o}^{d+1}\right)=1$.

In studying the integral (2.3), it is useful to distinguish between regular and irregular points of $W(\mathfrak{o})$. Let $\mathcal{U}_{1}$ denote the subvariety of $\mathbb{A}^{d}$ defined by the set of polynomials $F_{\rho}(\mathbf{Y})$ over $\mathfrak{o}$. We write $\mathbb{F}_{q}$ for the residue class field $\mathfrak{o} / \mathfrak{p}$. The reduction of $\mathfrak{U}_{1}$ modulo $\mathfrak{p}$ is denoted by $\overline{\mathcal{U}_{1}}$. We call a point $\mathbf{a} \in\left(\mathbb{F}_{q}^{d}\right)^{*}$, and any $\mathbf{y} \in W(\mathfrak{o})$ mapping onto a, regular if $\mathbf{a}$ is not an $\mathbb{F}_{q^{-}}$rational point of $\overline{\mathcal{U}_{1}}$. A functional $w \in \operatorname{Hom}_{\mathfrak{o}}(\mathfrak{g}, \mathfrak{o})^{*}$ and the representations associated to the Kirillov orbits of the images of $w$ in $\operatorname{Hom}_{\mathfrak{o}}\left(\mathfrak{g}, \mathfrak{o} / \mathfrak{p}^{n}\right)^{*}$, $n \in \mathbb{N}$, are said to be regular, if the co-ordinate vector $\mathbf{y} \in W(\mathfrak{o})$ corresponding to $w$ is regular. Points, functionals and representations which are not regular are called irregular. 
2.2. Adjoint versus co-adjoint action. In the special case where $\mathfrak{g}$ is an $\mathfrak{o}$-Lie lattice such that $\mathfrak{k} \otimes_{\mathfrak{o}} \mathfrak{g}$ is semisimple, one can use the Killing form, or a scaled version of it, to translate between co-adjoint orbits and adjoint orbits. This has some technical benefits when using the orbit method, as illustrated in Sections 4 and 5 .

Let $\mathfrak{g}$ be an $\mathfrak{o}$-lattice such that $\mathfrak{k} \otimes_{\mathfrak{o}} \mathfrak{g}$ is semisimple, and suppose that $m$ is permissible for $\mathfrak{g}$ so that the $m$ th principal congruence sublattice $\mathfrak{g}^{m}=\mathfrak{p}^{m} \mathfrak{g}$ is saturable and potent. At the level of the Lie algebra $\mathfrak{k} \otimes_{\mathfrak{o}} \mathfrak{g}$, the Killing form $\kappa$ is non-degenerate and thus provides an isomorphism $\iota$ of $\mathfrak{k}$-vector spaces between $\mathfrak{k} \otimes_{\mathfrak{o}} \mathfrak{g}$ and its dual space $\operatorname{Hom}_{\mathfrak{k}}\left(\mathfrak{k} \otimes_{\mathfrak{o}}\right.$ $\mathfrak{g}, \mathfrak{k})$. Moreover, this isomorphism is $G$-equivariant for any $G \leq \operatorname{Aut}\left(\mathfrak{k} \otimes_{\mathfrak{o}} \mathfrak{g}\right)$.

At the level of the $\mathfrak{o}$-Lie lattices $\mathfrak{g}$ and $\mathfrak{g}^{m}$, the situation is more intricate, because the restriction of $\kappa$, or a scaled version $\kappa_{0}$ of it, may not be non-degenerate over $\mathfrak{o}$. Typically, the pre-image of $\operatorname{Hom}_{\mathfrak{o}}(\mathfrak{g}, \mathfrak{o}) \hookrightarrow \operatorname{Hom}_{\mathfrak{k}}\left(\mathfrak{k} \otimes_{\mathfrak{o}} \mathfrak{g}, \mathfrak{k}\right)$ under the $\mathfrak{k}$-isomorphism $\iota_{0}: \mathfrak{k} \otimes_{\mathfrak{o}} \mathfrak{g} \rightarrow \operatorname{Hom}_{\mathfrak{k}}\left(\mathfrak{k} \otimes_{\mathfrak{o}} \mathfrak{g}, \mathfrak{k}\right)$ induced by $\kappa_{0}$ is an $\mathfrak{o}$-sublattice of $\mathfrak{k} \otimes_{\mathfrak{o}} \mathfrak{g}$ containing $\mathfrak{g}$ as a sublattice of finite index. For instance, if $\mathfrak{g}$ is a simple Lie algebra of Chevalley type, then it is natural to work with the normalised Killing form $\kappa_{0}$ which is related to the ordinary Killing form $\kappa$ by the equation $2 h^{\vee} \kappa_{0}=\kappa$. Here $h^{\vee}$ denotes the dual Coxeter number; e.g., the dual Coxeter number for $\mathfrak{s l}_{n}$ is $h^{\vee}=n$.

Irrespective of the detailed analysis required to translate carefully between adjoint and co-adjoint orbits, we obtain from the general discussion in [3, Section 5] a useful description of the parameters $\sigma(\mathfrak{g})$ and $\rho(\mathfrak{g})$, which were introduced in Section 2.1. Indeed, they can be computed in terms of centraliser dimensions as follows:

$$
\begin{aligned}
\operatorname{dim}_{\mathfrak{k}}\left(\mathfrak{k} \otimes_{\mathfrak{o}} \mathfrak{g}\right)-2 \sigma(\mathfrak{g}) & =\max \left\{\operatorname{dim}_{\mathfrak{k}} \mathrm{C}_{\mathfrak{k} \otimes_{\mathfrak{o}} \mathfrak{g}}(x) \mid x \in\left(\mathfrak{k} \otimes_{\mathfrak{o}} \mathfrak{g}\right) \backslash\{0\}\right\}, \\
\operatorname{dim}_{\mathfrak{k}}\left(\mathfrak{k} \otimes_{\mathfrak{o}} \mathfrak{g}\right)-2 \rho(\mathfrak{g}) & =\min \left\{\operatorname{dim}_{\mathfrak{k}} \mathrm{C}_{\mathfrak{k} \otimes_{\mathfrak{o}} \mathfrak{g}}(x) \mid x \in\left(\mathfrak{k} \otimes_{\mathfrak{o}} \mathfrak{g}\right) \backslash\{0\}\right\}
\end{aligned}
$$

2.3. General bounds for the abscissa of convergence. In this section we derive general bounds for the abscissae of convergence of zeta functions of compact $p$-adic analytic groups. We start by proving Theorem 1.1 which was stated in the introduction.

Proof of Theorem 1.1. Roughly speaking, the idea is that systematically overestimating the size of orbits in the co-adjoint action leads to a Dirichlet series $\psi_{\text {low }}(s)$ which converges at least as well as $\zeta_{\mathrm{G}^{m}}(s)$ and hence provides a lower bound for $\alpha\left(\mathrm{G}^{m}\right)$. Similarly, consistently underestimating the size of orbits leads to a Dirichlet series which converges no better than $\zeta_{\mathrm{G}^{m}}(s)$ and hence provides an upper bound for $\alpha\left(\mathrm{G}^{m}\right)$. For this we use the description of $\zeta_{\mathrm{G}^{m}}(s)$ in (2.1).

First we derive the lower bound for $\alpha\left(\mathrm{G}^{m}\right)$. Lemma 2.1, in conjunction with the definition of $\rho$, implies that $\left|\mathfrak{g}^{m}: \operatorname{Rad}(\omega)\right| \leq q^{2 \rho n}$ for all $n \in \mathbb{N}_{0}$ and $\omega \in \operatorname{Irr}_{n}\left(\mathfrak{g}^{m}\right)$. Clearly, the Dirichlet series

$$
\psi_{\text {low }}(s):=\sum_{n \in \mathbb{N}_{0}} \sum_{\omega \in \operatorname{Irr}_{n}\left(\mathfrak{g}^{m}\right)} q^{-\rho n(s+2)}
$$

converges better than $\zeta_{\mathrm{G}^{m}}(s)$ and it suffices to show that the abscissa of convergence of $\psi_{\text {low }}(s)$ is equal to $(d-2 \rho) \rho^{-1}$. Indeed, this can easily be read off from the precise 
formula

$$
\begin{aligned}
\psi_{\text {low }}(s) & =1+\sum_{n \in \mathbb{N}}\left(1-q^{-d}\right) q^{d n} q^{-\rho n(s+2)} \\
& =1+\left(1-q^{-d}\right) q^{(d-2 \rho)-\rho s}\left(1-q^{(d-2 \rho)-\rho s}\right)^{-1} \\
& =\left(1-q^{-2 \rho-\rho s}\right)\left(1-q^{(d-2 \rho)-\rho s}\right)^{-1}
\end{aligned}
$$

The argument for deriving the upper bound is essentially the same, but with a little extra twist. Recall that $W(\mathfrak{o})=\left(\mathfrak{o}^{d}\right)^{*}$. Similarly as in [3, Section 3.1] we consider a map $\nu: W(\mathfrak{o}) \rightarrow\left(\mathbb{N}_{0} \cup\{\infty\}\right)^{\lfloor d / 2\rfloor}$ which maps $\mathbf{y} \in W(\mathfrak{o})$ to the tuple $\mathbf{a}=\left(a_{1}, \ldots, a_{\lfloor d / 2\rfloor}\right)$ such that

(i) $a_{1} \leq \ldots \leq a_{\lfloor d / 2\rfloor}$ and

(ii) the elementary divisors of the anti-symmetric matrix $\mathcal{R}(\mathbf{y})$ are precisely $\mathfrak{p}^{a_{1}}, \ldots$, $\mathfrak{p}^{a\lfloor d / 2\rfloor}$, each counted with multiplicity 2 , and one further divisor $\mathfrak{p}^{\infty}$ if $d$ is odd.

The definition of $\sigma$ ensures that by forming the composition of $\nu$ with the projection $\left(a_{1}, \ldots, a_{\lfloor d / 2\rfloor}\right) \mapsto\left(a_{1}, \ldots, a_{\sigma}\right)$ we obtain a map $\nu_{\text {res }}: W(\mathfrak{o}) \rightarrow \mathbb{N}_{0}^{\sigma}$. Clearly, $\nu_{\text {res }}$ is continuous and hence locally constant. Since $W(\mathfrak{o})$ is compact, this implies that the image of $\nu_{\text {res }}$ is finite. From Lemma 2.1 we deduce that there is a constant $c \in \mathbb{N}_{0}$ such that for all $n \in \mathbb{N}_{0}$ and $\omega \in \operatorname{Irr}_{n}\left(\mathfrak{g}^{m}\right)$ we have $\left|\mathfrak{g}^{m}: \operatorname{Rad}(\omega)\right| \geq q^{2 \sigma n-c}$. Now a similar calculation as above gives the desired upper bound for $\alpha\left(\mathrm{G}^{m}\right)$.

Remark. (1) According to [16, Corollary 4.5], the abscissa of convergence $\alpha(G)$ is an invariant of the commensurability class of $G$. Thus Theorem 1.1 provides a tool for bounding the abscissa of convergence of the zeta function of any FAb compact $p$-adic analytic group; see Corollary 2.3 .

(2) The algebraic argument given in the proof of Theorem 1.1 admits a geometric interpretation based on the integral formula in Proposition 2.2, To obtain the lower bound one assumes that all points are 'regular', to obtain the lower bound that all points are as 'irregular' as possible.

For 'semisimple' compact $p$-adic analytic groups, the lower bound in Theorem 1.1 specialises to a result first proved by Larsen and Lubotzky; see [16, Proposition 6.6]. We formulate our more general result in this setting. Recall that to any compact $p$-adic analytic group $G$ one associates a $\mathbb{Q}_{p}$-Lie algebra, namely $L(G):=\mathbb{Q}_{p} \otimes_{\mathbb{Z}_{p}} \mathfrak{h}$ where $\mathfrak{h}$ is the $\mathbb{Z}_{p}$-Lie lattice associated to any saturable open pro- $p$ subgroup $H$ of $G$. This Lie algebra is an invariant of the commensurability class of $G$. Suppose that $L(G)$ is semisimple. Then it decomposes as a sum $L(G)=S_{1} \oplus \ldots \oplus S_{r}$ of simple $\mathbb{Q}_{p}$-Lie algebras $S_{i}$. For each $i \in\{1, \ldots, r\}$ the centroid $\mathfrak{k}_{i}$ of $S_{i}$, viz. the ring of $S_{i}$-endomorphisms of $S_{i}$ with respect to the adjoint action, is a finite extension field of $\mathbb{Q}_{p}$, and $S_{i}$ is an absolutely simple $\mathfrak{k}_{i}$-Lie algebra. The fields $\mathfrak{k}_{i}$ embed into the completion $\mathbb{C}_{p}$ of an algebraic closure of $\mathbb{Q}_{p}$. The field $\mathbb{C}_{p}$ is, algebraically, isomorphic to the field $\mathbb{C}$ of complex numbers. Indeed, $\mathbb{C}_{p}$ and $\mathbb{C}$ are algebraically closed and have the same uncountable transcendence degree over $\mathbb{Q}$. Choosing an isomorphism between $\mathbb{C}_{p}$ and $\mathbb{C}$, we define $\mathfrak{L}:=\mathfrak{L}(G):=\mathbb{C} \otimes_{\mathbb{Q}_{p}} L(G)$ and $\mathfrak{S}_{i}:=\mathbb{C} \otimes_{\mathfrak{k}_{i}} S_{i}$ for $i \in\{1, \ldots, r\}$. We define $r_{\text {abs }}(G)$ and $\Phi_{\text {abs }}(G)$ to be the absolute rank and the absolute root system of $L(G)$; they are equal to the rank and the root system of the semisimple complex Lie algebra $\mathfrak{L}$. We denote by $h^{\vee}(\mathfrak{S})$ the dual Coxeter number of a simple complex Lie algebra $\mathfrak{S}$. 
Corollary 2.3. Let $G$ be a compact p-adic analytic group such that its associated $\mathbb{Q}_{p^{-}}$ Lie algebra $L(G)$ is semisimple and decomposes as described above. Then the abscissa of convergence of $\zeta_{G}(s)$ satisfies

$$
\frac{2 r_{\mathrm{abs}}(G)}{\left|\Phi_{\mathrm{abs}}(G)\right|} \leq \alpha(G) \leq \min _{i \in\{1, \ldots, r\}} \frac{\operatorname{dim}_{\mathbb{C}}\left(\mathfrak{S}_{i}\right)}{h^{\vee}\left(\mathfrak{S}_{i}\right)-1}-2 .
$$

Proof. By our remark, we may assume without loss of generality that $G=\exp (\mathfrak{g})$ is associated to a potent and saturable $\mathbb{Z}_{p}$-Lie lattice $\mathfrak{g}$. The lower bound for $\alpha(G)$ follows immediately from Theorem 1.1 and the equations (2.4) on noting that $r_{\text {abs }}(G)=$ $\operatorname{dim}(G)-2 \rho(\mathfrak{g})$ and $\left|\Phi_{\text {abs }}(G)\right|=2 \rho(\mathfrak{g})$.

It remains to establish the upper bound. Replacing $G$ by an open subgroup, if necessary, we may assume that $\mathfrak{g}=\mathfrak{s}_{1} \oplus \ldots \oplus \mathfrak{s}_{r}$ and $G=G_{1} \times \ldots \times G_{r}$, where for each $i \in\{1, \ldots, r\}$ the summand $\mathfrak{s}_{i}$ is a potent and saturable $\mathbb{Z}_{p}$-Lie lattice such that $\mathbb{Q}_{p} \otimes_{\mathbb{Z}_{p}} \mathfrak{s}_{i}$ is simple and $G_{i}=\exp \left(\mathfrak{s}_{i}\right)$. As $\zeta_{G}(s)=\prod_{i=1}^{r} \zeta_{G_{i}}(s)$, we have $\alpha(G)=\min _{i \in\{1, \ldots, r\}} \alpha\left(G_{i}\right)$ and it is enough to bound $\alpha\left(G_{i}\right)$ for each $i \in\{1, \ldots, r\}$.

Fix $i \in\{1, \ldots, r\}$ and write $\mathfrak{s}:=\mathfrak{s}_{i}$. As before, the centroid $\mathfrak{k}$ of $\mathbb{Q}_{p} \otimes_{\mathbb{Z}_{p}} \mathfrak{s}$ is a finite extension of $\mathbb{Q}_{p}$, and $\mathbb{Q}_{p} \otimes_{\mathbb{Z}_{p}} \mathfrak{s}$ is an absolutely simple $\mathfrak{k}$-Lie algebra. Without loss of generality we may regard $\mathfrak{s}$ as an $\mathfrak{o}$-Lie lattice, where $\mathfrak{o}$ is the ring of integers of $\mathfrak{k}$. Writing $\mathfrak{S}=\mathbb{C} \otimes_{\mathfrak{o}} \mathfrak{s}$, we deduce from Theorem 1.1 that it suffices to show: $\sigma(\mathfrak{s}) \geq h^{\vee}(\mathfrak{S})-1$. It is clear that $2 \sigma(\mathfrak{s})$ is greater or equal to the dimension of a non-zero co-adjoint orbit of $\mathfrak{S}$ of minimal dimension. According to [6, Section 5.8], every sheet of $\mathfrak{S}$ contains a unique nilpotent orbit, and the dimension of a minimal nilpotent orbit in $\mathfrak{S}$ is equal to $2 h^{\vee}(\mathfrak{S})-2$; see [20, Theorem 1]. It follows that $\sigma(\mathfrak{s}) \geq h^{\vee}(\mathfrak{S})-1$.

It is worth pointing out that the absolute rank and the size of the absolute root system of a semisimple Lie algebra grow proportionally at the same rate under restriction of scalars; hence, if $G$ is defined over an extension $\mathfrak{o}$ of $\mathbb{Z}_{p}$, then it is natural to work directly with the invariants of the Lie algebra over $\mathfrak{k}$, without descending to $\mathbb{Q}_{p}$. A similar remark applies to the upper bound in Corollary 2.3. For instance, for the family of special linear groups $\mathrm{SL}_{n}(\mathfrak{o}), n \in \mathbb{N}$, we obtain the estimates

$$
2 / n \leq \alpha\left(\mathrm{SL}_{n}(\mathfrak{o})\right) \leq n-1,
$$

reflecting the fact that $\mathfrak{s l}_{n}(\mathbb{C})$ has rank $n-1$, a root system of size $n^{2}-n$, dimension $n^{2}-1$ and dual Coxeter number $h^{\vee}\left(\mathfrak{s l}_{n}(\mathbb{C})\right)=n$. More generally, we note that Corollary 2.3 provides upper bounds for the abscissae of convergence of zeta functions of groups corresponding to classical Lie algebras which are linear in the rank. We further remark that for 'isotropic simple' compact $p$-adic analytic groups the abscissa of convergence is actually bounded from below by 1/15; see [16, Theorem 8.1].

Another consequence of Theorem 1.1 is recorded as Corollary 4.1 in Section 4.1 .

\section{ExPLiCIT FORMUlaE FOR $\mathrm{SL}_{2}(\mathfrak{o})$ AND ITS PRINCIPAL CONGRUENCE SUbGROUPS}

In this section we use the setup from Sections 2.1, 2.2] and [3, Section 5] to compute explicitly the zeta functions of 'permissible' principal congruence subgroups of the compact $p$-adic analytic group $\mathrm{SL}_{2}(\mathfrak{o})$, where $\mathfrak{o}$ denotes a compact discrete valuation ring of characteristic 0 . As before, we write $\mathfrak{p}$ for the maximal ideal of $\mathfrak{o}$; the characteristic and cardinality of the residue field $\mathfrak{o} / \mathfrak{p}$ are denoted by $p$ and $q$. We write $e\left(\mathfrak{o}, \mathbb{Z}_{p}\right)$ for the absolute ramification index of $\mathfrak{o}$. 
3.1. Principal congruence subgroups of $\mathrm{SL}_{2}(\mathfrak{o})$. Our aim in this section is to prove Theorem 1.2 which was stated in the introduction. It provides explicit formulae for the zeta functions of principal congruence subgroups $\mathrm{SL}_{2}^{m}(\mathfrak{o})$ for permissible $m$, with no restrictions if $p>2$ and for unramified $\mathfrak{o}$ if $p=2$.

Remark. In fact, our proof also supplies an explicit formula, if $p=2$ and $e\left(\mathfrak{o}, \mathbb{Z}_{2}\right)>1$, but this formula is not as concise as the ones stated in Theorem 1.2, It is noteworthy that in this special case the formula only depends on the ramification index $e\left(\mathfrak{o}, \mathbb{Z}_{2}\right)$, but not on the more specific isomorphism type of the ring $\mathfrak{o}$. It would be interesting to investigate what happens for 'semisimple' groups of higher dimensions; already for $\mathrm{SL}_{3}(\mathfrak{o})$ the matter remains to be resolved; cf. Section 5 .

Proof of Theorem 1.2. Let $m \in \mathbb{N}$ be permissible for $\mathfrak{s l}_{2}(\mathfrak{o})$. We need to compute the integral (2.3) over $\mathfrak{p} \times W(\mathfrak{o})$, where $W(\mathfrak{o})=\left(\mathfrak{o}^{3}\right)^{*}$. It is easy to write down the commutator matrix $\mathcal{R}(\mathbf{Y})$ for the $\mathfrak{o}$-Lie lattice $\mathfrak{s l}_{2}(\mathfrak{o})$, and one verifies immediately that $\rho=1$. Indeed, working with the standard $\mathfrak{o}$-basis $\mathbf{e}=\left(\begin{array}{ll}0 & 1 \\ 0 & 0\end{array}\right), \mathbf{f}=\left(\begin{array}{ll}0 & 0 \\ 1 & 0\end{array}\right), \mathbf{h}=\left(\begin{array}{cc}1 & 0 \\ 0 & -1\end{array}\right)$ of $\mathfrak{s l}_{2}(\mathfrak{o})$ one obtains

$$
\mathcal{R}(\mathbf{Y})=\left(\begin{array}{ccc}
0 & Y_{3} & -2 Y_{1} \\
-Y_{3} & 0 & 2 Y_{2} \\
2 Y_{1} & -2 Y_{2} & 0
\end{array}\right)
$$

In view of (3.1) we distinguish two cases.

First suppose that $p>2$. In this case it is easily seen that

$$
\max \left\{|f(\mathbf{y})|_{\mathfrak{p}} \mid f \in F_{1}(\mathbf{Y})\right\} \cup\left\{\left|x^{2}\right|_{\mathfrak{p}}\right\}=1 \quad \text { for all } x \in \mathfrak{p} \text { and } \mathbf{y} \in W(\mathfrak{o}) .
$$

Thus the integral (2.3) takes the form

$$
z_{\mathfrak{o}}(r, t)=\int_{(x, \mathbf{y}) \in \mathfrak{p} \times W(\mathfrak{o})}|x|_{\mathfrak{p}}^{t} d \mu(x, \mathbf{y}) .
$$

As

$$
\int_{x \in \mathfrak{p}}|x|_{\mathfrak{p}}^{s} d \mu(x)=\frac{\left(1-q^{-1}\right) q^{-1-s}}{1-q^{-1-s}}
$$

and $\mu(W(\mathfrak{o}))=1-q^{-3}$ we obtain

$$
z_{\mathfrak{o}}(r, t)=\frac{\left(1-q^{-1}\right) q^{-1-t}\left(1-q^{-3}\right)}{1-q^{-1-t}}
$$

so that, by Proposition 2.2 , we have

$$
\zeta_{\mathrm{SL}_{2}^{m}(\mathfrak{o})}(s)=q^{3 m}\left(1+\left(1-q^{-1}\right)^{-1} z_{\mathfrak{o}}(-s / 2-1, s-2)\right)=\frac{q^{3 m}\left(1-q^{-2-s}\right)}{1-q^{1-s}} .
$$

Now consider the exceptional case $p=2$. Put $e:=e\left(\mathfrak{o}, \mathbb{Z}_{2}\right)$. Defining

$$
W(\mathfrak{o})^{[j]}:= \begin{cases}\left\{\mathbf{y} \in W(\mathfrak{o}) \mid y_{3} \in \mathfrak{p}^{j} \backslash \mathfrak{p}^{j+1}\right\} & \text { if } 0 \leq j \leq e-1, \\ \left\{\mathbf{y} \in W(\mathfrak{o}) \mid y_{3} \in 2 \mathfrak{o}\right\} & \text { if } j=e,\end{cases}
$$

we write $W(\mathfrak{o})$ as a disjoint union $W(\mathfrak{o})=W(\mathfrak{o})^{[0]} \cup W(\mathfrak{o})^{[1]} \cup \ldots \cup W(\mathfrak{o})^{[e]}$. From (3.1) we see that, for all $x \in \mathfrak{p}^{i} \backslash \mathfrak{p}^{i+1}$ and $\mathbf{y} \in W(\mathfrak{o})^{[j]}$, where $i \in \mathbb{N}$ and $1 \leq j \leq e$,

$$
\max \left\{|f(\mathbf{y})|_{\mathfrak{p}} \mid f \in F_{1}(\mathbf{Y})\right\} \cup\left\{\left|x^{2}\right|_{\mathfrak{p}}\right\}=q^{-2 \min \{i, j, e\}} .
$$


Furthermore, we note that $\mu\left(W(\mathfrak{o})^{[0]}\right)=\left(1-q^{-1}\right), \mu\left(W(\mathfrak{o})^{[j]}\right)=\left(1-q^{-1}\right)\left(1-q^{-2}\right) q^{-j}$ for $1 \leq j \leq e-1$, and $\mu\left(W(\mathfrak{o})^{[e]}\right)=\left(1-q^{-2}\right) q^{-e}$. Thus the integral (2.3) takes the form

$$
\begin{aligned}
z_{\mathfrak{o}}(r, t)= & \mu\left(W(\mathfrak{o})^{[0]}\right) \int_{x \in \mathfrak{p}}|x|_{\mathfrak{p}}^{t} d \mu(x) \\
& +\sum_{j=1}^{e} \mu\left(W(\mathfrak{o})^{[j]}\right)\left(\sum_{i=1}^{j-1} q^{-2 i r} \int_{x \in \mathfrak{p}^{i} \backslash \mathfrak{p}^{i+1}}|x|_{\mathfrak{p}}^{t} d \mu(x)+q^{-2 j r} \int_{x \in \mathfrak{p}^{j}}|x|_{\mathfrak{p}}^{t} d \mu(x)\right)
\end{aligned}
$$

which in turn yields an explicit formula for $\zeta_{\mathrm{SL}_{2}^{m}(\mathfrak{o})}(s)$, again by Proposition 2.2.

In the special case $e=e\left(\mathfrak{o}, \mathbb{Z}_{2}\right)=1$ the resulting formula is as concise as for $p>2$ : indeed, we have

$$
z_{\mathfrak{o}}(r, t)=\left(\left(1-q^{-1}\right)+\left(1-q^{-2}\right) q^{-1-2 r}\right) \frac{\left(1-q^{-1}\right) q^{-1-t}}{1-q^{-1-t}}
$$

and consequently

$$
\zeta_{\mathrm{SL}_{2}^{m}(\mathfrak{o})}(s)=q^{3 m}\left(1+\left(1-q^{-1}\right)^{-1} z_{\mathfrak{o}}(-s / 2-1, s-2)\right)=\frac{q^{3 m}\left(q^{2}-q^{-s}\right)}{1-q^{1-s}} .
$$

Computer aided calculations suggest the following conjecture.

Conjecture 3.1. The zeta function of $\mathrm{SL}_{2}^{1}\left(\mathbb{Z}_{2}\right)$ is given by

$$
\zeta_{\mathrm{SL}_{2}^{1}\left(\mathbb{Z}_{2}\right)}(s)=\frac{2^{5}\left(2^{2}-2^{-s}\right)}{1-2^{1-s}}
$$

3.2. Clifford theory. We briefly recall some applications of basic Clifford theory to representation zeta functions. For more details we refer to [3, Section 7]. Let $G$ be a group, and $N \unlhd G$ with $|G: N|<\infty$. For $\vartheta \in \operatorname{Irr}(N)$, let $I_{G}(\vartheta)$ denote the inertia group of $\vartheta$ in $G$, and $\operatorname{Irr}(G, \vartheta)$ the set of all irreducible characters $\rho$ of $G$ such that $\vartheta$ occurs as an irreducible constituent of the restricted character $\operatorname{res}_{N}^{G}(\rho)$. One shows that, if $N$ admits only finitely many irreducible characters of any given degree, then so does $G$ and

$$
\zeta_{G}(s)=\sum_{\vartheta \in \operatorname{Irr}(N)} \vartheta(1)^{-s} \cdot\left|G: I_{G}(\vartheta)\right|^{-1-s} \zeta_{G, \vartheta}(s)
$$

where

$$
\zeta_{G, \vartheta}(s):=\vartheta(1)^{s}\left|G: I_{G}(\vartheta)\right|^{s} \sum_{\rho \in \operatorname{Irr}(G, \vartheta)} \rho(1)^{-s} .
$$

In the special case where $\vartheta$ extends to an irreducible character $\hat{\vartheta}$ of $I_{G}(\vartheta)$, there is an effective description of the elements $\rho \in \operatorname{Irr}(G, \vartheta)$, and one has $\zeta_{G, \vartheta}(s)=\zeta_{I_{G}(\vartheta) / N}(s)$. There are several basic sufficient criteria for the extendability of $\vartheta$; cf. [10, Chapter 19].

3.3. The group $\mathrm{SL}_{2}(\mathfrak{o})$. In this section we combine the Kirillov orbit method and basic Clifford theory to compute explicitly the zeta function of the compact $p$-adic analytic group $\mathrm{SL}_{2}(\mathfrak{o})$. The zeta function of the group $\mathrm{SL}_{2}(R)$, where $R$ is an arbitrary compact discrete valuation ring of odd residue characteristic, was first computed by Jaikin-Zapirain by means of a different approach; see [12, Theorem 7.5].

For our approach we assume that $p-2 \geq e$ where $e:=e\left(\mathfrak{o}, \mathbb{Z}_{p}\right)$; in particular, this implies $p>2$. Then the $\mathfrak{o}$-Lie lattice $\mathfrak{s l}_{2}^{1}(\mathfrak{o})$ and the corresponding pro- $p$ group $\mathrm{SL}_{2}^{1}(\mathfrak{o})$ 


\begin{tabular}{|c|l|l|l|l|}
\hline type & & no. of orbits & size of each orbit & total number \\
\hline \hline 0 & - & 1 & 1 & 1 \\
1 & regular & 1 & $q^{2}-1$ & $q^{2}-1$ \\
$2 \mathrm{a}$ & regular & $(q-1) / 2$ & $q^{2}+q$ & $\left(q^{2}-1\right) q / 2$ \\
$2 \mathrm{~b}$ & regular & $(q-1) / 2$ & $q^{2}-q$ & $(q-1)^{2} q / 2$ \\
\hline
\end{tabular}

TABLE 3.1. Orbits in $\mathfrak{s l}_{2}\left(\mathbb{F}_{q}\right)$ under conjugacy by $\mathrm{GL}_{2}\left(\mathbb{F}_{q}\right)$ where $q=p^{r}$

are potent and saturable; see [3, Proposition 2.3]. This means that the orbit method can be applied to describe the irreducible characters of $\mathrm{SL}_{2}^{1}(\mathfrak{o})$.

Write $G:=\mathrm{SL}_{2}(\mathfrak{o})$ and $N:=\mathrm{SL}_{2}^{1}(\mathfrak{o})$. Clifford theory, as indicated in Section 3.2, provides a framework to $\operatorname{link} \operatorname{Irr}(G)$ and $\operatorname{Irr}(N)$. Put $\mathfrak{g}:=\mathfrak{s l}_{2}(\mathfrak{o})$ and $\mathfrak{n}:=\mathfrak{s l}_{2}^{1}(\mathfrak{o})=\mathfrak{p g}$. The Kirillov orbit method links characters $\vartheta \in \operatorname{Irr}(N)$ to co-adjoint orbits of $N$ on $\operatorname{Hom}_{\mathfrak{o}}(\mathfrak{n}, \mathfrak{o})$. Choose a uniformiser $\pi$ of $\mathfrak{o}$. Via the $G$-equivariant isomorphism of $\mathfrak{o}$ modules $\mathfrak{g} \rightarrow \mathfrak{n}, x \mapsto \pi x$, we can link co-adjoint orbits on $\operatorname{Hom}_{\mathfrak{o}}(\mathfrak{n}, \mathfrak{o})$ to co-adjoint orbits on $\operatorname{Hom}_{\mathfrak{o}}(\mathfrak{g}, \mathfrak{o})$.

We follow closely the approach outlined in [3, Section 5], which uses the normalised Killing form to translate between the adjoint action of $G$ on $\mathfrak{g}$ and the co-adjoint action of $G$ on $\operatorname{Hom}_{\mathfrak{o}}(\mathfrak{g}, \mathfrak{o})$. The dual Coxeter number of $\mathfrak{s l}_{2}$ is $h^{\vee}=2$ so that the normalised Killing form

$$
\kappa_{0}: \mathfrak{g} \times \mathfrak{g} \rightarrow \mathfrak{o}, \quad \kappa_{0}(x, y)=\left(2 h^{\vee}\right)^{-1} \operatorname{Tr}(\operatorname{ad}(x) \operatorname{ad}(y))
$$

has the structure matrix

$$
\left[\kappa_{0}(\cdot, \cdot)\right]_{(\mathbf{h}, \mathbf{e}, \mathbf{f})}=\left(\begin{array}{lll}
2 & 0 & 0 \\
0 & 0 & 1 \\
0 & 1 & 0
\end{array}\right)
$$

with respect to the basis

$$
\mathbf{h}=\left(\begin{array}{cc}
1 & 0 \\
0 & -1
\end{array}\right), \quad \mathbf{e}=\left(\begin{array}{ll}
0 & 1 \\
0 & 0
\end{array}\right), \quad \mathbf{f}=\left(\begin{array}{ll}
0 & 0 \\
1 & 0
\end{array}\right) .
$$

As $p>2$, the form $\kappa_{0}$ is 'non-degenerate' over $\mathfrak{o}$ and induces a $G$-equivariant isomorphism of $\mathfrak{o}$-modules $\mathfrak{g} \rightarrow \operatorname{Hom}_{\mathfrak{o}}(\mathfrak{g}, \mathfrak{o}), x \mapsto \kappa_{0}(x, \cdot)$. We obtain a $G$-equivariant commutative diagram

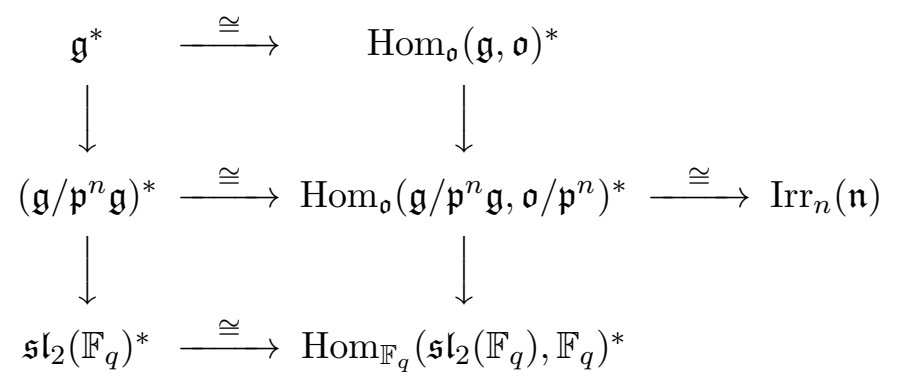

where the last row is obtained by reduction modulo $\mathfrak{p}$ and we have used the isomorphism $\mathfrak{o} / \mathfrak{p} \cong \mathbb{F}_{q}$. Following the approach taken in [3, Section 7], we are interested in the orbits and centralisers of elements $x \in \mathfrak{g}$ and their reductions $\bar{x}$ modulo $\mathfrak{p}$ under the adjoint action of $G$.

In order to apply Clifford theory, we require an overview of the elements in $\mathfrak{s l}_{2}\left(\mathbb{F}_{q}\right)$ up to conjugacy under the group $\mathrm{GL}_{2}\left(\mathbb{F}_{q}\right)$. We distinguish four different types, labelled $0,1,2 \mathrm{a}, 2 \mathrm{~b}$. The total number of elements of each type and the isomorphism types of their centralisers in $\mathrm{SL}_{2}\left(\mathbb{F}_{q}\right)$ are summarised in Tables 3.1 and 3.2, see Appendix $\mathrm{A}$ for a short discussion. We remark that in this particular case all elements are regular. 


\begin{tabular}{|c|l|l|}
\hline type & & centraliser in $\mathrm{SL}_{2}\left(\mathbb{F}_{q}\right)$ \\
\hline \hline 0 & - & $\mathrm{SL}_{2}\left(\mathbb{F}_{q}\right)$ \\
1 & regular & $\mu_{2}\left(\mathbb{F}_{q}\right) \times \mathbb{F}_{q}^{+} \cong C_{2} \times C_{p}^{r}$ \\
$2 \mathrm{a}$ & regular & $\mathbb{F}_{q}^{*} \cong C_{q-1}$ \\
$2 \mathrm{~b}$ & regular & $\operatorname{ker}\left(N_{\mathbb{F}_{q^{2}} \mid \mathbb{F}_{q}}\right) \cong C_{q+1}$ \\
\hline
\end{tabular}

TABLE 3.2. Centralisers in $\mathrm{SL}_{2}\left(\mathbb{F}_{q}\right)$ of elements of $\mathfrak{s l}_{2}\left(\mathbb{F}_{q}\right)$ where $q=p^{r}$

Corollary 7.6 in [3] provides

Lemma 3.2. Let $x \in \mathfrak{s l}_{2}(\mathfrak{o})^{*}$, and let $\bar{x} \in \mathfrak{s l}_{2}\left(\mathbb{F}_{q}\right)^{*}$ denote the reduction of $x$ modulo $\mathfrak{p}$. Then

$$
\mathrm{C}_{\mathrm{SL}_{2}(\mathfrak{o})}(\bar{x})=\mathrm{C}_{\mathrm{SL}_{2}(\mathfrak{o})}(x) \mathrm{SL}_{2}^{1}(\mathfrak{o}) .
$$

Remark. Alternatively, a direct argument shows that under the conjugation action by $\mathrm{GL}_{2}(\mathfrak{o})$ the elements of $\mathfrak{s l}_{2}(\mathfrak{o})^{*}$ fall into orbits represented by matrices of the form

$$
\begin{aligned}
& \left(\begin{array}{cc}
0 & 1 \\
\pi^{n} & \nu
\end{array}\right) \text { with } n \in \mathbb{N} \cup\{\infty\} \text { and } \nu \in \mathfrak{o} \backslash \mathfrak{p}, \\
& \left(\begin{array}{cc}
\lambda & 0 \\
0 & -\lambda
\end{array}\right) \text { with } \lambda \in \mathfrak{o} \backslash \mathfrak{p}, \\
& \left(\begin{array}{ll}
0 & 1 \\
\mu & 0
\end{array}\right) \text { with } \mu \in \mathfrak{o} \backslash \mathfrak{p} \text {, not a square modulo } \mathfrak{p} \text {. }
\end{aligned}
$$

A short computation shows that the centralisers of these matrices in $\mathrm{SL}_{2}(\mathfrak{o})$ are, respectively,

$$
\begin{gathered}
\left\{\left(\begin{array}{cc}
a & b \\
\pi^{n} \nu b & a
\end{array}\right) \mid a, b \in \mathfrak{o} \text { with } a^{2}-\pi^{n} \nu b^{2}=1\right\} \\
\left\{\left(\begin{array}{cc}
a & 0 \\
0 & b
\end{array}\right) \mid a, b \in \mathfrak{o} \text { with } a b=1\right\} \\
\left\{\left(\begin{array}{cc}
a & b \\
\mu b & a
\end{array}\right) \mid a, b \in \mathfrak{o} \text { with } a^{2}-\mu b^{2}=1\right\} .
\end{gathered}
$$

We may assume that $x$ is one of the listed representatives. The centraliser of $\bar{x}$ in $\mathrm{SL}_{2}\left(\mathbb{F}_{q}\right)$ has a similar form as that of $x$; cf. our discussion above. Aided by Hensel's lemma, one successfully lifts any given element $g_{0} \in \mathrm{C}_{\mathrm{SL}_{2}(\mathfrak{o})}(\bar{x})$ to an element $g \in$ $\mathrm{C}_{\mathrm{SL}_{2}(\mathfrak{o})}(\bar{x})$.

In any case, if $\vartheta \in \operatorname{Irr}(N)$ is represented by the adjoint orbit of $\left.x\right|_{n}:=x+\mathfrak{p}^{n} \mathfrak{g} \in$ $\left(\mathfrak{g} / \mathfrak{p}^{n} \mathfrak{g}\right)^{*}$, we deduce that $\mathrm{C}_{G}(x) N=\mathrm{C}_{G}\left(\left.x\right|_{n}\right) N=\mathrm{C}_{G}(\bar{x})$, hence

$$
I_{G}(\vartheta)=\mathrm{C}_{G}(\bar{x}) \quad \text { and } \quad I_{G}(\vartheta) / N \cong \mathrm{C}_{\mathrm{SL}_{2}\left(\mathbb{F}_{q}\right)}(\bar{x}) .
$$

The isomorphism types of these groups are given by Table 3.2, where $\mu_{2}\left(\mathbb{F}_{q}\right)$ is the group of square roots of unity in $\mathbb{F}_{q}^{*}$, we denote by $\mathbb{F}_{q}^{+}$the additive group of the field $\mathbb{F}_{q}$, and $\operatorname{ker}\left(N_{\mathbb{F}_{q^{2}} \mid \mathbb{F}_{q}}\right)$ is the multiplicative group of norm-1 elements in $\mathbb{F}_{q^{2}} \mid \mathbb{F}_{q}$.

Note that, if $\vartheta$ is of type $2 \mathrm{a}$ or $2 \mathrm{~b}$, the inertia group quotient $I_{G}(\vartheta) / N$ has order coprime to $p$. This implies that $\vartheta$ can be extended to a character $\hat{\vartheta}$ of $I_{G}(\vartheta)$. If $\vartheta$ is of type 1, the inertia group is a Sylow pro- $p$ subgroup of $\mathrm{SL}_{2}(\mathfrak{o})$, and we can draw the same conclusion based on [1, Theorem 2.3]: being an algebra group, the character degrees of $I_{G}(\vartheta)$ are powers of $q$, and since $\left|I_{G}(\vartheta): N\right|=q$, Lemma 7.4 in [3] shows that $\vartheta$ extends to a character $\hat{\vartheta}$ of $I_{G}(\vartheta)$. (If $2 e<p-1$, the Sylow pro- $p$ subgroup of $\mathrm{SL}_{2}(\mathfrak{o})$ corresponds to a potent and saturable o-Lie lattice; cf. [14. Hence one can apply [3, Corollary 3.2] to show that its character degrees are powers of $q$.) Since the inertia group quotients are abelian, it follows that in all cases $\zeta_{G, \vartheta}(s)=\zeta_{I_{G}(\vartheta) / N}(s)=\left|I_{G}(\vartheta): N\right|$. 
The final task is to connect the formula

$$
\zeta_{G}(s)=\sum_{\vartheta \in \operatorname{Irr}(N)} \vartheta(1)^{-s} \cdot\left|G: I_{G}(\vartheta)\right|^{-1-s}\left|I_{G}(\vartheta): N\right|,
$$

resulting from (3.2), and the explicit formula for $\zeta_{N}(s)=\sum_{\vartheta \in \operatorname{Irr}(N)} \vartheta(1)^{-s}$ which we obtained in Section 3.1. We parameterise the non-trivial characters $\operatorname{in} \operatorname{Irr}(N)$ by means of the affine cone $W(\mathfrak{o})=\left(\mathfrak{o}^{3}\right)^{*}$, uniformly for all levels, as in the integral formula (2.3). The affine cone $W(\mathfrak{o})$ decomposes as a disjoint union of subsets $W(\mathfrak{o})^{[1]}, W(\mathfrak{o})^{[2 \mathrm{a}]}$ and $W(\mathfrak{o})^{[2 \mathrm{~b}]}$ corresponding to representations of types $1,2 a$ and $2 b$. According to our explicit element count modulo $\mathfrak{p}$ (see Table 3.1), we have $\mu\left(W(\mathfrak{o})^{[1]}\right)=q^{-1}\left(1-q^{-2}\right)$, $\mu\left(W(\mathfrak{o})^{[2 \mathrm{a}]}\right)=\left(1-q^{-2}\right) / 2$ and $\mu\left(W(\mathfrak{o})^{[2 \mathrm{~b}]}\right)=\left(1-q^{-1}\right)^{2} / 2$. With this preparation we may write

$$
\zeta_{N}(s)=1+q^{3}\left(\mu\left(W(\mathfrak{o})^{[1]}\right)+\mu\left(W(\mathfrak{o})^{[2 \mathrm{a}]}\right)+\mu\left(W(\mathfrak{o})^{[2 \mathrm{~b}]}\right)\right)\left(1-q^{1-s}\right)^{-1},
$$

and this yields

$$
\begin{aligned}
\zeta_{\mathrm{SL}_{2}(\mathfrak{o})}(s)=\zeta_{\mathrm{SL}_{2}\left(\mathbb{F}_{q}\right)}(s)+q^{3}\left(\mu\left(W(\mathfrak{o})^{[1]}\right)\left(\left(q^{2}-1\right) / 2\right)^{-1-s} 2 q+\right. \\
\mu\left(W(\mathfrak{o})^{[2 \mathrm{a}]}\right)\left(q^{2}+q\right)^{-1-s}(q-1)+ \\
\left.\mu\left(W(\mathfrak{o})^{[2 \mathrm{~b}]}\right)\left(q^{2}-q\right)^{-1-s}(q+1)\right)\left(1-q^{1-s}\right)^{-1} \\
=1+X_{1}+\frac{q-3}{2} X_{2}+2 X_{3}+\frac{q-1}{2} X_{4}+2 X_{5}+ \\
\left(1-q X_{1}\right)^{-1}\left(4 q X_{2} X_{5}+\frac{q^{2}-1}{2} X_{1} X_{4}+\frac{(q-1)^{2}}{2} X_{1} X_{2}\right)
\end{aligned}
$$

where $X_{1}=q^{-s}, X_{2}=(q+1)^{-s}, X_{3}=((q+1) / 2)^{-s}, X_{4}=(q-1)^{-s}$ and $X_{5}=$ $((q-1) / 2)^{-s}$. The last formula is in agreement with [12, Theorem 7.5].

It is worth pointing out that $\zeta_{\mathrm{SL}_{2}(\mathfrak{o})}(s)$, unlike $\zeta_{\mathrm{SL}_{2}^{1}(\mathfrak{o})}(s)$, cannot be written as a rational function in $q^{-s}$. In [3, Theorem A], we establish in a rather general context local functional equations for the zeta functions associated to families of pro- $p$ groups, such as $\mathrm{SL}_{2}^{1}(\mathfrak{o})$; cf. Theorem 1.2. It would be very interesting if these could be meaningfully extended to the zeta functions of larger compact $p$-adic analytic groups, such as $\mathrm{SL}_{2}(\mathfrak{o})$.

Remark. It would be interesting to see if the use of Clifford theory, as explored in the current section, can also be employed to establish the conjectural formula for the zeta function of $\mathrm{SL}_{2}^{1}\left(\mathbb{Z}_{2}\right)$ stated in Conjecture 3.1. For this one would start from our analysis of the zeta function $\zeta_{\mathrm{SL}_{2}^{2}\left(\mathbb{Z}_{2}\right)}(s)$; cf. Theorem 1.2,

3.4. The group $\mathrm{SL}_{2}\left(\mathbb{Z}_{2}\right)$. The paper [17] contains an explicit construction of the irreducible representations of the group $\mathrm{SL}_{2}\left(\mathbb{Z}_{2}\right)$. This is achieved by decomposing Weil representations associated to binary quadratic forms and by considering tensor products of certain components of such Weil representations. To complement (3.5) we record the following immediate consequence of the work in [17].

Theorem 3.3. We have

$$
\zeta_{\mathrm{SL}_{2}\left(\mathbb{Z}_{2}\right)}(s)=\frac{\left(4-2^{1-s}-5 \cdot 2^{1-2 s}+2^{1-3 s}\right)+3^{-s}\left(28+2^{1-s}-5 \cdot 2^{1-2 s}+2^{1-3 s}\right)}{1-2^{1-s}} .
$$

Proof. It follows by inspection of the classification results in [17, pp. 522-524] that the continuous irreducible characters of $G:=\mathrm{SL}_{2}\left(\mathbb{Z}_{2}\right)$ all have degrees of the form $2^{i}$ or $3 \cdot 2^{i}$, 
for $i \in \mathbb{N}_{0}$. Concerning 2-power-degree characters, one has

$$
r_{1}(G)=4, \quad r_{2}(G)=6, \quad r_{2^{2}}(G)=2, \quad \text { and } \quad r_{2^{i}}(G)=3 \cdot 2^{i-2} \text { for } i \geq 3 .
$$

This yields

$$
\sum_{i=0}^{\infty} r_{2^{i}}(G)\left(2^{-s}\right)^{i}=\frac{4-2 \cdot\left(2^{-s}\right)-10 \cdot 2^{-2 s}+2 \cdot 2^{-3 s}}{1-2 \cdot 2^{-s}} .
$$

The numbers of characters of degree $3 \cdot 2^{i}$, for $i \in \mathbb{N}_{0}$, are given by

$$
r_{3}(G)=28, \quad r_{3 \cdot 2}(G)=58, \quad r_{3 \cdot 2^{2}}(G)=106, \quad \text { and } \quad r_{3 \cdot 2^{i}}(G)=107 \cdot 2^{i-2} \text { for } i \geq 3 .
$$

Indeed, for $i \geq 3$ the characters of degree $3 \cdot 2^{i}$ come from levels $i+1, i+2, i+3$ and $i+4$, with contributions from these levels of $2^{i-2}, 2^{i-1}, 5 \cdot 2^{i+1}$ and $2^{i+4}$ characters, respectively. One checks that $2^{i-2}+2^{i-1}+5 \cdot 2^{i+1}+2^{i+4}=107 \cdot 2^{i-2}$, as claimed. This yields

$$
\sum_{i=0}^{\infty} r_{3 \cdot 2^{i}}(G)\left(3^{-s}\right)\left(2^{-s}\right)^{i}=3^{-s}\left(\frac{28+2 \cdot 2^{-s}-10 \cdot 2^{-2 s}+2 \cdot 2^{-3 s}}{1-2 \cdot 2^{-s}}\right) .
$$

Combining (3.6) and (3.7) yields the claimed expression.

The results in 17] do not indicate how to compute the zeta function of $\mathrm{SL}_{2}(\mathfrak{o})$ for extension rings $\mathfrak{o}$ of $\mathbb{Z}_{2}$. In view of our earlier computations it would be particularly interesting to consider the case where $\mathfrak{o}$ is an unramified extension of $\mathbb{Z}_{2}$.

\section{ExPlicit Formulae For SUbGRoups of QUATERNiON GROUPS $\mathrm{SL}_{1}(\mathfrak{D})$}

The aim of this section is to provide a setup for computing the zeta function of the norm one group $\mathrm{SL}_{1}(\mathfrak{D})$ of a central division algebra $\mathfrak{D}$ over a $\mathfrak{p}$-adic field $\mathfrak{k}$. Our approach leads to immediate consequences in the special case where the Schur index $\ell$ of $\mathfrak{D}$ over $\mathfrak{k}$ is a prime number. Explicit formulae are given for the zeta functions of norm one groups of non-split quaternion algebras; see Theorem 1.3 below.

4.1. General division algebras. Let $\mathfrak{D}$ denote a central division algebra of Schur index $\ell \geq 2$ over a $\mathfrak{p}$-adic field $\mathfrak{k}$. Let $\mathfrak{o}$ denote the valuation ring in $\mathfrak{k}$, with maximal ideal $\mathfrak{p}$, and let $\mathfrak{R}$ denote the maximal compact subring of $\mathfrak{D}$, with maximal ideal $\mathfrak{P}$. Write $q$ and $p$ for the cardinality and characteristic of the residue field of $\mathfrak{o}$.

We consider the compact $p$-adic analytic group $G:=\mathrm{SL}_{1}(\mathfrak{D})=\mathrm{SL}_{1}(\mathfrak{R})$ of norm-1 elements in $\mathfrak{D}$ and its principal congruence subgroups $G_{m}:=\mathrm{SL}_{1}^{m}(\mathfrak{D})=\mathrm{SL}_{1}(\mathfrak{D}) \cap(1+$ $\left.\mathfrak{P}^{m}\right), m \in \mathbb{N}$. We remark that the resulting congruence filtration of $G$ is a refinement of the filtration that one would get from restriction of scalars to $\mathfrak{o}$ and defining congruence subgroups in terms of $\mathfrak{p}$; this justifies the slight difference in notation from Section 2, The group $G / G_{1}$ is isomorphic to the multiplicative group of norm-1 elements of $\mathfrak{R} / \mathfrak{P} \cong \mathbb{F}_{q^{\ell}}$ over $\mathfrak{o} / \mathfrak{p} \cong \mathbb{F}_{q}$, and hence cyclic of order $\left(q^{\ell}-1\right) /(q-1)$. Each of the quotients $G_{m} / G_{m+1}$, $m \in \mathbb{N}$, embeds into the additive group $\mathfrak{R} / \mathfrak{P} \cong \mathbb{F}_{q^{\ell}}$ and is thus an elementary abelian $p$-group. It follows that $G_{1}$ is the unique Sylow pro- $p$ subgroup of $G$. The k-Lie algebra associated to the group $\mathrm{SL}_{1}(\mathfrak{D})$ is $\mathfrak{s l}_{1}(\mathfrak{D})$, consisting of all trace- 0 elements of $\mathfrak{D}$.

To begin with, we derive the following consequence of Theorem 1.1 and its Corollary 2.3, which is based on the extra assumption that $\ell$ is prime. 
Corollary 4.1. Let $\mathfrak{D}$ be a central division algebra of prime Schur index $\ell$ over $\mathfrak{k}$. Then the abscissa of convergence of $\zeta_{\mathrm{SL}_{1}(\mathfrak{D})}(s)$ is equal to $2 / \ell=2 r_{\mathrm{abs}} /\left|\Phi_{\mathrm{abs}}\right|$, where $r_{\mathrm{abs}}$ is the absolute rank of $\mathfrak{s l}_{1}(\mathfrak{D})$ and $\Phi_{\text {abs }}$ denotes the absolute root system associated to $\mathfrak{s l}_{1}(\mathfrak{D})$.

We remark that a more complex argument shows that the conclusion of the corollary remains true even if the Schur index $\ell$ is not prime; see [16, Theorem 7.1].

Proof of Corollary 4.1. The absolute rank of $\mathfrak{s l}_{1}(\mathfrak{D})$ is $r_{\text {abs }}=\ell-1$, and the size of the absolute root system associated to $\mathfrak{s l}_{1}(\mathfrak{D})$ is $\left|\Phi_{\text {abs }}\right|=\ell^{2}-\ell$. Hence $2 / \ell=2 r_{\text {abs }} /\left|\Phi_{\text {abs }}\right|$. Clearly, $\operatorname{dim}_{\mathfrak{k}}\left(\mathfrak{s l}_{1}(\mathfrak{D})\right)=\ell^{2}-1$. Recall that the abscissa of convergence $\alpha\left(\mathrm{SL}_{1}(\mathfrak{D})\right)$ is a commensurability invariant of the group $\mathrm{SL}_{1}(\mathfrak{D})$. Thus, in view of Theorem 1.1 and the equations (2.4), it suffices to prove that $\operatorname{dim}_{\mathfrak{k}} \mathrm{C}_{\mathfrak{s l}}(\mathfrak{D})(x)=\ell-1$ for all $x \in \mathfrak{s l}_{1}(\mathfrak{D}) \backslash\{0\}$. Indeed, this will imply $\sigma\left(\mathfrak{s l}_{1}(\mathfrak{R})\right)=\rho\left(\mathfrak{s l}_{1}(\mathfrak{R})\right)=\ell(\ell-1) / 2$ and the result follows.

Let $x \in \mathfrak{s l}_{1}(\mathfrak{D}) \backslash\{0\}$. Then $\mathfrak{k}(x) \mid \mathfrak{k}$ is a non-trivial field extension, and the Centraliser Theorem for central simple algebras yields $\ell^{2}=|\mathfrak{D}: \mathfrak{k}|=|\mathfrak{k}(x): \mathfrak{k}|\left|\mathrm{C}_{\mathfrak{D}}(x): \mathfrak{k}\right|$. Since $\ell$ is assumed to be prime, this implies $\left|\mathrm{C}_{\mathfrak{D}}(x): \mathfrak{k}\right|=\ell$, and hence $\operatorname{dim}_{\mathfrak{k}} \mathrm{C}_{\mathfrak{s l}_{1}(\mathfrak{D})}(x)=\ell-1$.

As a step toward the explicit computation of the zeta functions of $G_{1}$ and $G$, we describe sufficient conditions for applying the Kirillov orbit method to capture the irreducible complex characters of the group $G_{1}$.

Proposition 4.2. Let $\mathfrak{D}$ be a central division algebra of Schur index $\ell$ over the $\mathfrak{p}$-adic field $\mathfrak{k}$. Let $\mathfrak{R}$ be the maximal compact subring of $\mathfrak{D}$, and suppose that $p \nmid \ell$, where $p$ denotes the residue field characteristic of $\mathfrak{k}$.

Then $G_{1}=\mathrm{SL}_{1}^{1}(\mathfrak{R})$ is an insoluble maximal p-adic analytic just infinite pro-p group. Furthermore, if $e\left(\mathfrak{k}, \mathbb{Q}_{p}\right) \ell<p-1$, then $G_{1}$ is potent and saturable.

Proof. It is known that $\operatorname{Aut}\left(\mathfrak{s l}_{1}(\mathfrak{D})\right) \cong \mathrm{PGL}_{1}(\mathfrak{D})$; see $\left[13\right.$, $\S \mathrm{XI}$ e]. The groups $\mathrm{SL}_{1}(\mathfrak{D})$ and $\mathrm{PGL}_{1}(\mathfrak{D})$ are, of course, closely related. Indeed, $Z:=\mathrm{Z}\left(\mathrm{GL}_{1}(\mathfrak{D})\right)=\mathfrak{k}^{*}$ and the norm map induces an isomorphism

$$
\mathrm{GL}_{1}(\mathfrak{D}) / Z \cdot \mathrm{SL}_{1}(\mathfrak{D}) \cong \mathfrak{k}^{*} /\left(\mathfrak{k}^{*}\right)^{\ell}
$$

Denote by $\mu_{\ell}\left(\mathfrak{k}^{*}\right)$ the finite subgroup of $\mathfrak{k}^{*}$ consisting of all elements whose order divides $\ell$. Since $p \nmid \ell$, reduction modulo $\mathfrak{p}$ maps $\mu_{\ell}\left(\mathfrak{k}^{*}\right) \leq \mathfrak{o}^{*}$ injectively onto the cyclic subgroup of order $\operatorname{gcd}(\ell, q-1)$ of $\mathbb{F}_{q}^{*}$. Moreover, $G_{1} \cap Z=G_{1} \cap \mu_{\ell}\left(\mathfrak{k}^{*}\right)=1$, and the order of $\mathfrak{k}^{*} /\left(\mathfrak{k}^{*}\right)^{\ell}$ is not divisible by $p$. Hence we see from the exact sequence

$$
1 \rightarrow \mu_{\ell}\left(\mathfrak{k}^{*}\right) \rightarrow \mathrm{SL}_{1}(\mathfrak{D}) \rightarrow \mathrm{PGL}_{1}(\mathfrak{D}) \rightarrow \mathfrak{k}^{*} /\left(\mathfrak{k}^{*}\right)^{\ell} \rightarrow 1
$$

that $G_{1}$ is isomorphic to a Sylow pro- $p$ subgroup of the compact group $\mathrm{PGL}_{1}(\mathfrak{D})$. It follows that $G_{1}$ is an insoluble maximal $p$-adic analytic just infinite pro- $p$ group; cf. [13, $\S$ III e].

Let $\mathfrak{K}$ be a splitting subfield of $\mathfrak{D}$, unramified and of degree $\ell$ over $\mathfrak{k}$. Then the $\mathfrak{K}$-algebra isomorphism $\mathfrak{K} \otimes_{\mathfrak{k}} \mathfrak{D} \cong \operatorname{Mat}_{\ell}(\mathfrak{K})$ provides an embedding of $G_{1}$ into a Sylow pro-p subgroup $S$ of $\mathrm{SL}_{\ell}(\mathfrak{O})$, where $\mathfrak{O}$ denotes the valuation ring of $\mathfrak{K}$. Suppose that $e \ell<p-1$, where $e=e\left(\mathfrak{k}, \mathbb{Q}_{p}\right)=e\left(\mathfrak{O}, \mathbb{Z}_{p}\right)$. From [15, III (3.2.7)] we conclude that $S$ is saturable. Now we conclude as in [14, Proof of Theorem 1.3] that $G_{1}$ is saturable. Moreover, $\gamma_{p-1}\left(G_{1}\right) \subseteq \gamma_{\ell \ell+1}\left(G_{1}\right)=G_{1}^{p}$ (cf. [18, $\left.\left.\S 1\right]\right)$ so that $G_{1}$ is potent. 
4.2. The quaternion case. In this section we consider the special case $\ell=2$, where $\mathfrak{D}$ is a non-split quaternion algebra over the $\mathfrak{p}$-adic field $\mathfrak{k}$, but some of the arguments below are equally relevant in the more general situation where $p \nmid \ell$. Concretely, we compute explicit formulae for the zeta functions of norm one quaternion groups $\mathrm{SL}_{1}(\mathfrak{D})=\mathrm{SL}_{1}(\mathfrak{R})$ and their principal congruence subgroups $\mathrm{SL}_{1}^{m}(\mathfrak{R})$, as stated in Theorem 1.3 in the introduction.

Remark. Similar formulae as the ones provided in Theorem 1.3 can be obtained for higher principal congruence subgroups $\mathrm{SL}_{1}^{m}(\mathfrak{R})$, even if the condition $e\left(\mathfrak{k}, \mathbb{Q}_{p}\right) \ell<p-1$ imposed in the theorem is not satisfied. Our computation can be carried out in a similar fashion whenever the orbit method can be applied.

It is a natural and interesting problem to compute explicit formulae for the zeta functions of norm one groups $\mathrm{SL}_{1}(\mathfrak{D})$ and their principal congruence groups, where the Schur index of $\mathfrak{D}$ over $\mathfrak{k}$ is greater than 2 . Another interesting group to consider would be $\mathrm{SL}_{2}(\mathfrak{R})$, where $\mathfrak{R}$ is the maximal compact subring of a non-split quaternion algebra $\mathfrak{D}$ over $\mathfrak{k}$.

Proof of Theorem 1.3. According to Proposition 4.2, the pro- $p$ group $G_{1}$ is saturable and potent. Our first aim is to compute an explicit formula for the zeta functions of the principal congruence subgroups $G_{m}=\mathrm{SL}_{1}^{m}(\mathfrak{R}), m \in \mathbb{N}$. Put $\mathfrak{g}:=\mathfrak{s l}_{1}(\mathfrak{R})$, and for $m \in \mathbb{N}$ let $\mathfrak{g}_{m}:=\mathfrak{s l}_{1}^{m}(\mathfrak{R})=\mathfrak{s l}_{1}(\mathfrak{R}) \cap \mathfrak{P}^{m}$ denote the $m$ th principal congruence Lie sublattice so that $G_{m}=\exp \left(\mathfrak{g}_{m}\right)$. Here $\mathfrak{o}$ denotes, as usual, the valuation ring of $\mathfrak{k}$, with maximal ideal $\mathfrak{p}=\pi \mathfrak{o}$ generated by a uniformiser $\pi$.

Since $p \neq 2$, we may choose $1, \mathbf{u}, \mathbf{v}, \mathbf{u v}$ as a standard basis for $\mathfrak{D}$ over $\mathfrak{k}$, where $\mathbf{u}^{2}=a \in \mathfrak{o}$ is not a square modulo $\mathfrak{p}, \mathbf{v}^{2}=\pi$ and $\mathbf{u v}=-\mathbf{v u}$. Writing $\mathbf{i}:=\frac{1}{2} \mathbf{u}, \mathbf{j}:=\frac{1}{2} \mathbf{v}$ and $\mathbf{k}:=\frac{1}{2} \mathbf{u v}$ we have

$$
[\mathbf{i}, \mathbf{j}]=\mathbf{k}, \quad[\mathbf{i}, \mathbf{k}]=a \mathbf{j}, \quad[\mathbf{j}, \mathbf{k}]=-\pi \mathbf{i} .
$$

An $\mathfrak{o}$-basis for $\mathfrak{g}_{1}$ is then given by $\pi \mathbf{i}, \mathbf{j}$, $\mathbf{k}$, with corresponding commutator matrix

$$
\mathcal{R}(\mathbf{Y})=\left(\begin{array}{ccc}
0 & \pi Y_{3} & a \pi Y_{2} \\
-\pi Y_{3} & 0 & -Y_{1} \\
-a \pi Y_{2} & Y_{1} & 0
\end{array}\right) .
$$

In view of Proposition 2.2, an argument similar as for the case $p=2$ and $e=1$ in Section 3.1 shows that

$$
\zeta_{\mathrm{SL}_{1}^{m}(\mathfrak{R})}(s)=q^{3(m-1)} \frac{q^{2}-q^{-s}}{1-q^{1-s}} \quad \text { for } m \in \mathbb{N} .
$$

Our next aim is to deduce a formula for the zeta function of the group $G=\mathrm{SL}_{1}(\mathfrak{R})$, using Clifford theory, similarly as in Section 3.3. For this it is useful to record the following intermediate formula which comes from a similar argument as in Section 3.1 .

$$
\zeta_{\mathrm{SL}_{1}^{1}(\mathfrak{R})}(s)=1+\left(\mu\left(W(\mathfrak{o})^{[1]}\right) q^{1-s}+\mu\left(W(\mathfrak{o})^{[2]}\right) q^{3}\right) \frac{1}{1-q^{1-s}},
$$

where $W(\mathfrak{o})^{[1]}=\left\{\mathbf{y} \in W(\mathfrak{o}) \mid y_{1} \in \mathfrak{o}^{*}\right\}$ and $W(\mathfrak{o})^{[2]}=\left\{\mathbf{y} \in W(\mathfrak{o}) \mid y_{1} \in \mathfrak{p}\right\}$ have Haar measure $\mu\left(W(\mathfrak{o})^{[1]}\right)=1-q^{-1}$ and $\mu\left(W(\mathfrak{o})^{[2]}\right)=q^{-1}\left(1-q^{-2}\right)$ respectively.

We continue to write $G=\mathrm{SL}_{1}(\mathfrak{R})$ and put $N:=G_{1}=\mathrm{SL}_{1}^{1}(\Re)$. Since $G / N$ is cyclic, any irreducible character $\vartheta$ of $N$ can a priori be extended to an irreducible character 
$\hat{\vartheta}$ of its inertia group $I_{G}(\vartheta)$. Thus, similarly as for the group $\mathrm{SL}_{2}(\mathfrak{o})$, the central task consists in describing the inertia groups in order to apply (3.4). We show below that

(i) $I_{G}(\vartheta)=G$, and hence $I_{G}(\vartheta) / N$ is cyclic of order $q+1$, if $\vartheta \in \operatorname{Irr}(G)$ corresponds to a co-adjoint orbit of a functional represented by an element of $W(\mathfrak{o})^{[1]}$,

(ii) $I_{G}(\vartheta)=\{1,-1\} N$, and hence $I_{G}(\vartheta) / N$ is cyclic of order 2 , if $\vartheta \in \operatorname{Irr}(G)$ corresponds to a co-adjoint orbit of a functional represented by an element of $W(\mathfrak{o})^{[2]}$.

We conclude the proof by applying Clifford theory as in Section 3.3. Putting together the general formula (3.4), the specific formula (4.3) and statements (i) and (ii), we deduce that

$$
\begin{aligned}
\zeta_{\mathrm{SL}_{1}(\mathfrak{R})}(s)= & \zeta_{C_{q+1}}(s)+\left(\mu\left(W(\mathfrak{o})^{[1]}\right) q^{1-s}(q+1)+\right. \\
& \left.\left.\mu\left(W(\mathfrak{o})^{(2)}\right) q^{3}((q+1) / 2)^{-1-s} 2\right)\right) \frac{1}{1-q^{1-s}} \\
= & \frac{(q+1)\left(1-q^{-s}\right)+4(q-1)((q+1) / 2)^{-s}}{1-q^{1-s}} .
\end{aligned}
$$

It remains to justify the assertions (i) and (ii). For this it is convenient to translate between the adjoint action of $G$ on $\mathfrak{g}$ and the co-adjoint action of $G$ on $\operatorname{Hom}_{\mathfrak{o}}(\mathfrak{g}, \mathfrak{o})$. From (4.1) one easily sees that the normalised Killing form $\kappa_{0}: \mathfrak{g} \times \mathfrak{g} \rightarrow \mathfrak{o}$ has the structure matrix

$$
\left[\kappa_{0}(\cdot, \cdot)\right]_{(\mathbf{i}, \mathbf{j}, \mathbf{k})}=\left(\begin{array}{ccc}
a & 0 & 0 \\
0 & \pi & 0 \\
0 & 0 & -a \pi
\end{array}\right)
$$

with respect to the basis $\mathbf{i}, \mathbf{j}, \mathbf{k}$. While $\kappa_{0}$ is degenerate over $\mathfrak{o}$, the form is non-degenerate over $\mathfrak{k}$ and induces a bijective linear map $\iota_{0}: \mathfrak{s l}_{1}(\mathfrak{D}) \rightarrow \operatorname{Hom}_{\mathfrak{k}}\left(\mathfrak{s l}_{1}(\mathfrak{D}), \mathfrak{k}\right)$. We have $\mathfrak{g}_{-1}:=\mathfrak{s l}_{1}(\mathfrak{R}) \cap \mathfrak{P}^{-1}=\iota_{0}^{-1}\left(\operatorname{Hom}_{\mathfrak{o}}(\mathfrak{g}, \mathfrak{o})\right)$ and $\mathfrak{g}_{-2}:=\mathfrak{s l}_{1}(\mathfrak{R}) \cap \mathfrak{P}^{-2}=\iota_{0}^{-1}\left(\operatorname{Hom}_{\mathfrak{o}}\left(\mathfrak{g}_{1}, \mathfrak{o}\right)\right)$. The decomposition $\operatorname{Hom}_{\mathfrak{o}}\left(\mathfrak{g}_{1}, \mathfrak{o}\right)^{*}=W(\mathfrak{o})^{[1]} \cup W(\mathfrak{o})^{[2]}$ corresponds to the decomposition

$$
\mathfrak{g}_{-2}^{*}=\left(\mathfrak{g}_{-2} \backslash \mathfrak{g}_{-1}\right) \cup\left(\mathfrak{g}_{-1} \backslash \mathfrak{g}\right) .
$$

The action of $G / N$ on quotients $\mathfrak{g}_{m} / \mathfrak{g}_{m+1}$ of successive terms in the congruence filtration of $\mathfrak{g}$ is described in [18, $\S 1$ ] and we will use a compatible notation as far as practical. The division algebra $\mathfrak{D}$ contains an unramified extension $\mathfrak{K}=\mathfrak{k}(\mathbf{i})$ of degree 2 over $\mathfrak{k}$ which is normalised by the uniformiser $\Pi:=\mathbf{j}$. Let $F$ denote the residue field of $\mathfrak{K}$, and let $\Phi$ denote the group of roots of unity in $\mathfrak{K}$. Thus $F \cong \mathbb{F}_{q^{2}}$ and $\Phi \cup\{0\}$ is a set of representatives for the elements of $F$. Observe that $N$ is complemented in $G$ by the subgroup $H$ of $\Phi$ consisting of all roots of unity which are of norm 1 over $\mathfrak{k}$ : we have $G=H \ltimes N$. Accordingly, we will think of $G / N \cong H$ as the group of elements in the finite field $F$ which have norm 1 over $f:=\mathfrak{o} / \mathfrak{p} \cong \mathbb{F}_{q}$. Every element of $\mathfrak{R}$ has a unique power series expansion in $\Pi$ with coefficients in $\Phi \cup\{0\}$. For each $m \in \mathbb{N}$ this induces an embedding $\eta_{m}: \mathfrak{g}_{m} / \mathfrak{g}_{m+1} \hookrightarrow F$; we denote the image of $\eta_{m}$ by $F(m)$. If $2 \nmid m$ then $F(m)=F$, and if $2 \mid m$ then $F(m)=\left\{x \in F \mid \operatorname{Tr}_{F \mid f}(x)=0\right\}$.

Clearly, for each $m \in \mathbb{N}$ the action of $G$ on $F(m)$ by conjugation factors through $N$ and is therefore determined by the action of $H$. The latter is given by the explicit formula

$$
x^{h}=h^{1-q^{m}} \cdot x, \quad \text { for } x \in F(m) \text { and } h \in H .
$$

This finishes our preparations and we turn to the proof of assertions (i) and (ii) above.

First we consider a character $\vartheta \in \operatorname{Irr}(N)$ corresponding to the co-adjoint orbit of $\omega \in \operatorname{Irr}\left(\mathfrak{g}_{1}\right)$, where $\omega$ is represented by an element of $W(\mathfrak{o})^{[1]}$ and has level $n$, say; cf. Section 2.1. Then the inertia group $I_{G}(\vartheta)$ is equal to $C N$, where $C:=\mathrm{C}_{G}\left(x+\mathfrak{g}_{2 n-2}\right)$ 
for a suitable $x \in \mathfrak{g}_{-2} \backslash \mathfrak{g}_{-1}$; see (4.4). Here $\mathfrak{g}_{0}:=\mathfrak{g}$ if $n=1$. We claim that $C N=G$, justifying (i). For this it is enough to prove that $H$ centralises a suitable $N$-conjugate of $x$. Since $H$ is a subgroup of the multiplicative group of the field $\mathfrak{K}$, it suffices to show that $x$ is $N$-conjugate to an element of $\mathfrak{K}$. For this we construct recursively a sequence $x_{0}, x_{1}, \ldots$ of $N$-conjugates of $x$ such that $x_{i} \equiv \lambda_{i} \pi^{-1} \mathbf{i}$ modulo $\mathfrak{g}_{i-1}$ with $\lambda_{i} \in \mathfrak{o} \backslash \mathfrak{p}$ for each index $i$. From the construction one sees that the sequence converges and its limit is an $N$-conjugate of $x$ in $\mathfrak{K}$. Since $x \in \mathfrak{g}_{-2} \backslash \mathfrak{g}_{-1}$, we can take $x_{0}:=x$. Now suppose that $i \in \mathbb{N}_{0}$ and that

$$
x_{i}=\lambda_{i} \pi^{-1} \mathbf{i}+\mu \mathbf{j}+\nu \mathbf{k} \quad \text { with } \lambda_{i}, \mu, \nu \in \mathfrak{o}, \lambda_{i} \notin \mathfrak{p} \text { and } \mu \mathbf{j}+\nu \mathbf{k} \in \mathfrak{P}^{i-1}
$$

is an $N$-conjugate of $x$. Then $x_{i+1}:=z^{-1} x_{i} z$ with $z:=1-\lambda_{i}^{-1} \pi\left(\nu \mathbf{j}-a^{-1} \mu \mathbf{k}\right) \in$ $1+\mathfrak{P}^{i+1} \subseteq N$ is an $N$-conjugate of $x$ and satisfies the desired congruence, modulo $\mathfrak{g}_{i}$,

$$
\begin{aligned}
x_{i+1} & \equiv\left(1+\lambda_{i}^{-1} \pi\left(\nu \mathbf{j}+a^{-1} \mu \mathbf{k}\right)\right)\left(\lambda_{i} \pi^{-1} \mathbf{i}+(\mu \mathbf{j}+\nu \mathbf{k})\right)\left(1-\lambda_{i}^{-1} \pi\left(\nu \mathbf{j}+a^{-1} \mu \mathbf{k}\right)\right) \\
& \equiv \lambda_{i} \pi^{-1} \mathbf{i}+(\mu \mathbf{j}+\nu \mathbf{k})+\left[\nu \mathbf{j}+a^{-1} \mu \mathbf{k}, \mathbf{i}\right] \\
& \equiv \lambda_{i} \pi^{-1} \mathbf{i} .
\end{aligned}
$$

Finally we consider a character $\vartheta \in \operatorname{Irr}(N)$ corresponding to the co-adjoint orbit of $\omega \in \operatorname{Irr}\left(\mathfrak{g}_{1}\right)$, where $\omega$ is represented by an element of $W(\mathfrak{o})^{[2]}$ and has level $n$, say. Then the inertia group $I_{G}(\vartheta)$ is equal to $C N$, where $C:=\mathrm{C}_{G}\left(x+\mathfrak{g}_{2 n-2}\right)$ for a suitable $x \in \mathfrak{g}_{-1} \backslash \mathfrak{g}$; see (4.4). We claim that $C N=\{1,-1\} N$, justifying (ii). Since $x \in \mathfrak{g}_{-1}$, we have $\{1,-1\} N \subseteq C N \subseteq \mathrm{C}_{G}(x+\mathfrak{g})=\mathrm{C}_{H}(x+\mathfrak{g}) N$. Hence it suffices to prove that $\mathrm{C}_{H}(x+\mathfrak{g})=\{1,-1\}$. Indeed, multiplication by $\pi$ provides an $H$-equivariant isomorphism $\mathfrak{g}_{-1} / \mathfrak{g} \rightarrow \mathfrak{g}_{1} / \mathfrak{g}_{2}$. The action of $h \in H$ by conjugation on $\mathfrak{g}_{1} / \mathfrak{g}_{2}$ corresponds to multiplication by $h^{1-q}$ on $F(1)$; to carry out the multiplication $h$ is considered as a element of the residue field $F$ with norm 1 in $f$, as described above. The group $H$ has order $q+1$, hence the kernel of $H \rightarrow H, h \mapsto h^{1-q}$ is equal to $\{1,-1\}$. It follows that $\mathrm{C}_{H}(\tilde{x})=\{1,-1\}$ for any non-zero element $\tilde{x} \in F(1)$.

\section{Principal congruence subgroups $\mathrm{OF} \mathrm{SL}_{3}(\mathfrak{o})$ For unramified o of Residue FIELD CHARACTERISTIC 3}

Let $\mathfrak{o}$ be a compact discrete valuation ring of characteristic 0 and residue field characteristic $p$. Except for the special case $p=3$, Theorem $\mathrm{E}$ in [3] provides an explicit universal formula for the zeta functions of principal congruence subgroups of $\mathrm{SL}_{3}(\mathfrak{o})$. By a different approach, the same formula and indeed a formula for the group $\mathrm{SL}_{3}(\mathfrak{o})$ itself are derived in [4]. In this section we complement the generic formulae by proving Theorem 1.4 which was stated in the introduction. This theorem provides explicit formulae for the zeta functions of principal congruence subgroups of $\mathrm{SL}_{3}(\mathfrak{o})$, where $\mathfrak{o}$ has residue characteristic 3 and is unramified over $\mathbb{Z}_{3}$.

Residue field characteristic 3 was excluded from [3, Theorem E], whose proof is based on a geometric description of the variety of irregular elements in the 8-dimensional Lie algebra $\mathfrak{s l}_{3}(\mathfrak{k})$. This description breaks down when the map $\beta: \mathfrak{g l}_{3} \rightarrow \mathfrak{s l}_{3}, \mathbf{x} \mapsto$ $\mathbf{x}-\operatorname{Tr}(\mathbf{x}) / 3$ used in [3, Section 6.1] displays bad reduction modulo $\mathfrak{p}$. Moreover, the translation of the relevant $\mathfrak{p}$-adic integral via the normalised Killing form becomes more technical. In the present paper we restrict our attention to unramified extensions $\mathfrak{o}$ of $\mathbb{Z}_{3}$ for simplicity. Indeed, the results in Section 3.1 suggest that analogous formulae 
which are to cover the general case, including ramification, are likely to become rather cumbersome to write down.

The method we employ is algebraic and somewhat closer to the approach taken in [4]. In fact, the arguments which we shall supply can be employed mutatis mutandis in the generic case $p \neq 3$ and hence give an alternative, less geometric derivation of the formula provided in [3, Theorem E]. Moreover, our calculations illustrate the algebraic meaning of the $\mathfrak{p}$-adic formalism developed and applied in [3].

5.1. Let $\mathfrak{o}$ be unramified over $\mathbb{Z}_{p}$ with residue field $\mathfrak{o} / \mathfrak{p} \cong \mathbb{F}_{q}$ of characteristic $p=3$. Throughout the section we will continue to write $p$ as far as convenient, while keeping the concrete value $p=3$ in mind. We remark that $p$ is also a uniformiser for $\mathfrak{o}$, because $\mathfrak{o}$ be unramified over $\mathbb{Z}_{p}$, and we will use $p$ instead of the symbol $\pi$. Let $T_{\mathfrak{o}}=\{0\} \cup \mu_{q-1}(\mathfrak{o})$ denote the set of Teichmüller representatives for $\mathfrak{o}$, which projects bijectively onto the residue field of $\mathfrak{o}$. More generally, for any $l \in \mathbb{N}$ we fix

$$
T_{\mathfrak{o}}(l):=\left\{\sum_{j=0}^{l-1} t_{j} p^{j} \mid t_{j} \in T_{\mathfrak{o}} \text { for } 0 \leq j<l\right\}
$$

as a set of representatives for $\mathfrak{o} / \mathfrak{p}^{l}$. As usual, $\mathfrak{k}$ denotes the field of fractions of $\mathfrak{o}$.

Let $m \in \mathbb{N}$. Proposition 2.3 in [3] shows that $m$ is permissible for $\mathfrak{s l}_{3}(\mathfrak{o})$. Thus the zeta function of the $m$ th principal congruence subgroup $\mathrm{SL}_{3}^{m}(\mathfrak{o})$ is given by the formula

$$
\zeta_{\mathrm{SL}_{3}^{m}(\mathfrak{o})}(s)=q^{8 m}\left(1+\left(1-q^{-1}\right)^{-1} z_{\mathfrak{o}}(-s / 2-1,3 s-3)\right),
$$

which results from Proposition 2.2 on setting $d=\operatorname{dim}_{\mathfrak{k}} \mathfrak{s l}_{3}(\mathfrak{k})=8$ and $\rho=2^{-1}\left(\operatorname{dim}_{\mathfrak{k}} \mathfrak{s l}_{3}(\mathfrak{k})-\right.$ $\left.r_{\text {abs }}\left(\mathfrak{s l}_{3}(\mathfrak{k})\right)\right)=3$; see (2.4). As indicated in Section 2.1, the integral $z_{\mathfrak{o}}(r, t)$ is intimately linked to the elementary divisors of the commutator matrix $\mathcal{R}(\mathbf{y})$ for $\mathfrak{s l}_{3}(\mathfrak{o})$, evaluated at points $\mathbf{y} \in W(\mathfrak{o})=\left(\mathfrak{o}^{8}\right)^{*}$. We observe that the commutator matrix $\mathcal{R}(\mathbf{y})$, at $\mathbf{y} \in W(\mathfrak{o})$, has Witt normal form

$$
\left(\begin{array}{ccccccccc}
0 & 1 & & & & & & \\
-1 & 0 & & & & & & \\
& & 0 & 1 & & & & \\
& -1 & 0 & & & & & \\
& & & & 0 & p^{a} & & \\
& & & & -p^{a} & 0 & & \\
& & & & & 0 & 0 \\
& & & & & & 0 & 0
\end{array}\right)
$$

so that all the information is condensed in a single parameter $a=a(\mathbf{y}) \in \mathbb{N}_{0} \cup\{\infty\}$.

The integral $z_{\mathfrak{o}}(r, t)$ in (2.3) is defined so that it performs integration over the space $\mathfrak{p} \times \operatorname{Hom}_{\mathfrak{o}}\left(\mathfrak{s l}_{3}(\mathfrak{o}), \mathfrak{o}\right)^{*}$ with respect to a particular choice of coordinate system $(x, \mathbf{y}) \in$ $\mathfrak{p} \times W(\mathfrak{o})$. The normalised Killing form $\kappa_{0}$ of $\mathfrak{s l}_{3}(\mathfrak{k})$ is related to the ordinary Killing form $\kappa: \mathfrak{s l}_{3}(\mathfrak{k}) \times \mathfrak{s l}_{3}(\mathfrak{k}) \rightarrow \mathfrak{k}$ by the equation $\kappa=2 h^{\vee} \kappa_{0}=6 \kappa_{0}$; see Section 2.2. In [3, Section 6], we provided the structure matrix of the normalised Killing form $\kappa_{0}$ with respect to the basis

$$
\begin{aligned}
& \mathbf{h}_{12}=\left(\begin{array}{lll}
1 & & \\
& -1 & \\
& & 0
\end{array}\right), \quad \mathbf{h}_{23}=\left(\begin{array}{lll}
0 & & \\
& 1 & \\
& & -1
\end{array}\right),
\end{aligned}
$$

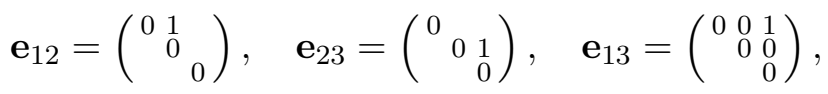

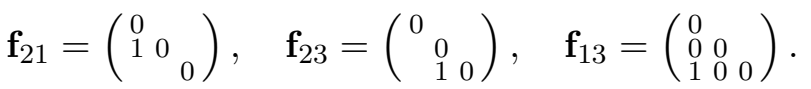

This matrix has determinant 3. Thus the form $\kappa_{0}$ induces a bijective linear map $\iota_{0}$ : $\mathfrak{s l}_{3}(\mathfrak{k}) \rightarrow \operatorname{Hom}_{\mathfrak{k}}\left(\mathfrak{s l}_{3}(\mathfrak{k}), \mathfrak{k}\right)$, but becomes more intricate at the level of $\mathfrak{o}$-lattices, due to the 
residue field characteristic 3. Indeed, the pre-image of $\operatorname{Hom}_{\mathfrak{o}}\left(\mathfrak{s l}_{3}(\mathfrak{o}), \mathfrak{o}\right)$ under $\iota_{0}$ is the o-lattice

$$
\Lambda:=\iota_{0}^{-1}\left(\operatorname{Hom}_{\mathfrak{o}}\left(\mathfrak{s l}_{3}(\mathfrak{o}), \mathfrak{o}\right)\right)=\bigcup_{u \in T_{\mathfrak{o}}(1)}\left(u\left(\frac{2}{3} \mathbf{h}_{12}+\frac{1}{3} \mathbf{h}_{23}\right)+\mathfrak{s l}_{3}(\mathfrak{o})\right)
$$

Thus we have $p \Lambda \leq \mathfrak{s l}_{3}(\mathfrak{o}) \leq \Lambda$ with $\left|\mathfrak{s l}_{3}(\mathfrak{o}): p \Lambda\right|=q^{7}$ and $\left|\Lambda: \mathfrak{s l}_{3}(\mathfrak{o})\right|=q$. We pull back the integral $z_{\mathfrak{o}}(r, t)$ over $\mathfrak{p} \times\left(\operatorname{Hom}_{\mathfrak{o}}\left(\mathfrak{s l}_{3}(\mathfrak{o}), \mathfrak{o}\right)\right)^{*}$ to an integral over $\mathfrak{p} \times \Lambda^{*}$, taking into account the Jacobi factor $|3|_{\mathfrak{p}}=q^{-1}$. Dividing the new region of integration with respect to the second factor into cosets modulo $p \Lambda$, we write

$$
z_{\mathfrak{o}}(r, t)=\mathcal{S}_{1}(r, t)+\mathcal{S}_{2}(r, t),
$$

where the two summands correspond to the complementary subregions of integration $\mathfrak{p} \times\left(\mathfrak{s l}_{3}(\mathfrak{o}) \backslash p \Lambda\right)$ and $\mathfrak{p} \times\left(\Lambda \backslash \mathfrak{s l}_{3}(\mathfrak{o})\right)$ respectively. We show in Sections 5.2 and 5.3 that these summands are given by the formulae

$$
\begin{aligned}
& \mathcal{S}_{1}(r, t)=\left(q^{4}+q^{3}-q-1\right) z_{\mathfrak{o}}^{[3]}(r, t)+\left(q^{6}-q^{4}-q^{3}+q\right) z_{\mathfrak{o}}^{[2]}(r, t)+\left(q^{7}-q^{6}\right) z_{\mathfrak{o}}^{[0]}(r, t), \\
& \mathcal{S}_{2}(r, t)=(q-1)\left(\left(q^{2}+q+1\right) q^{2} z_{\mathfrak{o}}^{[1]}(r, t)+\left(q^{7}-\left(q^{2}+q+1\right) q^{2}\right) z_{\mathfrak{o}}^{[0]}(r, t)\right),
\end{aligned}
$$

where

$$
\begin{aligned}
& z_{\mathfrak{o}}^{[0]}(r, t)=\int_{(x, \mathbf{y}) \in \mathfrak{p} \times \mathfrak{p}(8)}|x|^{t} d \mu(x, \mathbf{y}), \\
& z_{\mathfrak{o}}^{[1]}(r, t)=\int_{(x, \mathbf{y}) \in \mathfrak{p} \times \mathfrak{p}(8)}|x|^{t}\left\|\left\{y_{1}, y_{2}, y_{3}, x\right\}\right\|_{\mathfrak{p}}^{2 r} d \mu(x, \mathbf{y}), \\
& z_{\mathfrak{o}}^{[2]}(r, t)=q^{-2 r} z_{\mathfrak{o}}^{[0]}(r, t), \\
& z_{\mathfrak{o}}^{[3]}(r, t)=\int_{(x, \mathbf{y}) \in \mathfrak{p} \times \mathfrak{p}(8)}|x|^{t}\left\|\left\{p y_{1}, p y_{2}, y_{3}, x\right\}\right\|_{\mathfrak{p}}^{2 r} d \mu(x, \mathbf{y}) .
\end{aligned}
$$

In [3, Section 6.1] it is shown that

$$
z_{\mathfrak{o}}^{[0]}(r, t)=\frac{q^{-9-t}\left(1-q^{-1}\right)}{1-q^{-1-t}}
$$

and

$$
z_{\mathfrak{o}}^{[1]}(r, t)=\frac{q^{-9-2 r-t}\left(1-q^{-4-t}\right)\left(1-q^{-1}\right)}{\left(1-q^{-4-2 r-t}\right)\left(1-q^{-1-t}\right)} .
$$

The latter may be obtained from the formula

$$
z_{\mathfrak{o}}^{[1]}(r, t)=\sum_{(l, n) \in \mathbb{N}^{2}}\left(1-q^{-1}\right) q^{-n} m_{l}^{[1]} q^{-n t-2 \min \{l, n\} r},
$$

with

$$
m_{l}^{[1]}:=\mu\left(\left\{\mathbf{y} \in \mathfrak{p}^{(8)} \mid \max \left\{\left|y_{1}\right|_{\mathfrak{p}},\left|y_{2}\right|_{\mathfrak{p}},\left|y_{3}\right|_{\mathfrak{p}}\right\}=q^{-l}\right\}\right)=\left(1-q^{-3}\right) q^{-3 l-5},
$$

using the fact that

$$
\sum_{(l, n) \in \mathbb{N}^{2}} X_{1}^{l} X_{2}^{n} X_{3}^{\min \{l, n\}}=\frac{X_{1} X_{2} X_{3}\left(1-X_{1} X_{2}\right)}{\left(1-X_{1} X_{2} X_{3}\right)\left(1-X_{1}\right)\left(1-X_{2}\right)} .
$$


Clearly, (5.5) implies that

$$
z_{\mathfrak{o}}^{[2]}(r, t)=\frac{q^{-9-2 r-t}\left(1-q^{-1}\right)}{1-q^{-1-t}} .
$$

This formula, too, may be written as a sum

$$
z_{\mathfrak{o}}^{[2]}(r, t)=\sum_{(l, n) \in \mathbb{N}^{2}}\left(1-q^{-1}\right) q^{-n} m_{l}^{[2]} q^{-n t-2 \min \{l, n\} r},
$$

with

$$
m_{l}^{[2]}:=\mu\left(\left\{\left.\mathbf{y} \in \mathfrak{p}^{(8)}|| p\right|_{\mathfrak{p}}=q^{-l}\right\}\right)= \begin{cases}q^{-8} & \text { if } l=1 \\ 0 & \text { if } l \geq 2 .\end{cases}
$$

It remains to compute $z_{\mathfrak{o}}^{[3]}(r, t)$. We have

$$
z_{\mathfrak{o}}^{[3]}(r, t)=\sum_{(l, n) \in \mathbb{N}^{2}}\left(1-q^{-1}\right) q^{-n} m_{l}^{[3]} q^{-n t-2 \min \{l, n\} r}
$$

where

$$
\begin{aligned}
m_{l}^{[3]} & :=\mu\left(\left\{\mathbf{y} \in \mathfrak{p}^{(8)} \mid \max \left\{\left|p y_{1}\right|_{\mathfrak{p}},\left|p y_{2}\right|_{\mathfrak{p}},\left|y_{3}\right|_{\mathfrak{p}}\right\}=q^{-l}\right\}\right) \\
& = \begin{cases}\left(1-q^{-1}\right) q^{-8} & \text { if } l=1, \\
\left(1-q^{-3}\right) q^{-3-3 l} & \text { if } l \geq 2 .\end{cases}
\end{aligned}
$$

Using (5.8) this gives

$$
\begin{aligned}
\mathcal{Z}_{\mathfrak{o}}^{[3]}(r, t)= & \left(1-q^{-1}\right)\left(m_{1}^{[3]}-\left(1-q^{-3}\right) q^{-6}\right) \sum_{n \in \mathbb{N}} q^{-(1+t) n-2 r} \\
& +\left(1-q^{-1}\right)\left(1-q^{-3}\right) q^{-3} \sum_{(l, n) \in \mathbb{N}^{2}} q^{-3 l-(1+t) n-2 \min \{l, n\} r} \\
= & -\left(1-q^{-1}\right)\left(1-q^{-2}\right) q^{-7-2 r-t}\left(1-q^{-1-t}\right)^{-1} \\
& +\left(1-q^{-1}\right)\left(1-q^{-4-t}\right) q^{-7-t-2 r}\left(1-q^{-1-t}\right)^{-1}\left(1-q^{-4-t-2 r}\right)^{-1} \\
= & \frac{\left(1-q^{-1}\right) q^{-9-t-2 r}\left(1-q^{-2-t}+q^{-2-t-2 r}-q^{-4-t-2 r}\right)}{\left(1-q^{-1-t}\right)\left(1-q^{-4-t-2 r}\right)}
\end{aligned}
$$

A short computation, based on (5.2), (5.3) and (5.4), now yields the explicit formula for the zeta function of $\mathrm{SL}_{3}^{m}(\mathfrak{o})$, stated in Theorem 1.4.

The remainder of this section is devoted to an algebraic justification of the equations (5.4). It would be interesting to derive a geometric explanation, more similar to the argument in [3, Section 6.1] treating the generic case.

5.2. First we will derive the formula given for the summand $\mathcal{S}_{1}(r, t)$ in (5.4). For this we decompose $\mathfrak{s l}_{3}(\mathfrak{o}) \backslash p \Lambda$ into cosets modulo $p \Lambda$, or equivalently the finite Lie algebra $\mathfrak{s l}_{3}\left(\mathbb{F}_{q}\right)$ into cosets modulo its centre. As $p=3$, the centre of $\mathfrak{s l}_{3}\left(\mathbb{F}_{q}\right)$ is the 1-dimensional subalgebra $\mathbb{F}_{q} \overline{\mathbf{z}}$, spanned by the reduction modulo $\mathfrak{p}$ of $\mathbf{z}:=\mathbf{h}_{12}-\mathbf{h}_{23}$, viz. the subalgebra of scalar matrices over $\mathbb{F}_{q}$. An overview of the orbits in $\mathfrak{s l}_{3}\left(\mathbb{F}_{q}\right)$ under the adjoint action of $\mathrm{GL}_{3}\left(\mathbb{F}_{q}\right)$ is provided in Table 5.1; see Appendix $\mathrm{C}$ for a short discussion. The second column indicates whether the corresponding elements are regular or irregular, as defined at the end of Section 2.1. The corresponding total number of cosets modulo $\mathbb{F}_{q} \overline{\mathbf{z}}$, given in the last column of the table, is obtained upon division by $q$. A short calculation 
confirms that the sizes of the orbits listed in Table 5.1 add up to $q^{7}=\left|\mathfrak{s l}_{3}\left(\mathbb{F}_{q}\right): \mathbb{F}_{q} \overline{\mathbf{z}}\right|$, as wanted.

\begin{tabular}{|c|l|l|l|l|}
\hline type & & no. of orbits & size of each orbit & total number modulo $\mathbb{F}_{q} \mathbf{z}$ \\
\hline \hline $\mathrm{A}$ & & $q$ & 1 & 1 \\
$\mathrm{~B}$ & irregular & $q$ & $\left(q^{3}-1\right)\left(q^{2}-1\right) q$ & $\left(q^{3}-1\right)\left(q^{2}-1\right) q$ \\
$\mathrm{C}$ & irregular & $q$ & $\left(q^{3}-1\right)(q+1)$ & $\left(q^{3}-1\right)(q+1)$ \\
$\mathrm{D}$ & regular & $(q-1) q / 6$ & $\left(q^{2}+q+1\right)(q+1) q^{3}$ & $\left(q^{3}-1\right)(q+1) q^{3} / 6$ \\
$\mathrm{E}$ & regular & $(q-1) q / 2$ & $\left(q^{3}-1\right) q^{3}$ & $\left(q^{3}-1\right)(q-1) q^{3} / 2$ \\
$\mathrm{~F}$ & regular & $(q-1) q / 3$ & $\left(q^{2}-1\right)(q-1) q^{3}$ & $\left(q^{2}-1\right)(q-1)^{2} q^{3} / 3$ \\
\hline
\end{tabular}

TABLE 5.1. Adjoint orbits in $\mathfrak{s l}_{3}\left(\mathbb{F}_{q}\right)$ under the action of $\mathrm{GL}_{3}\left(\mathbb{F}_{q}\right), q \equiv_{3} 0$

The equation for $\mathcal{S}_{1}(r, t)$ in (5.4) indicates that $z_{\mathfrak{o}}^{[0]}(r, t)$ is the correct integral for the types $\mathrm{D}, \mathrm{E}, \mathrm{F}$, which cover the regular elements modulo $p$. It remains to link the contributions to the summand $\mathcal{S}_{1}(r, t)$ by irregular elements belonging to cosets of types $\mathrm{B}$ and $\mathrm{C}$ to the integrals $z_{\mathfrak{o}}^{[2]}(r, t)$ and $z_{\mathfrak{o}}^{[3]}(r, t)$, respectively.

5.2.1. Let us consider first elements belonging to cosets modulo $p \Lambda$ of type $\mathrm{C}$ and work out the integral around such elements which results from pulling the original integral $z_{\mathfrak{o}}(r, t)$ over $\mathfrak{p} \times\left(\operatorname{Hom}_{\mathfrak{o}}\left(\mathfrak{s l}_{3}(\mathfrak{o}), \mathfrak{o}\right)\right)^{*}$ back to $\mathfrak{p} \times \Lambda^{*}$. A typical coset of type $\mathrm{C}$ is $\mathbf{a}_{0}+p \Lambda$, where $\mathbf{a}_{0}:=\left(\begin{array}{lll}0 & 1 & 0 \\ 0 & 0 & 0 \\ 0 & 0 & 0\end{array}\right)$, and each coset has measure $\mu(p \Lambda)=q^{-7}$. As indicated earlier, the determinant of the Jacobi matrix associated to $\iota_{0}: \Lambda \rightarrow \operatorname{Hom}_{\mathfrak{o}}\left(\mathfrak{s l}_{3}(\mathfrak{o}), \mathfrak{o}\right)$ is 3 and thus contributes another factor $|3|_{\mathfrak{p}}=q^{-1}$. The integral over $\mathfrak{p} \times(\mathbf{a}+p \Lambda)$ with Jacobi factor $q^{-1}$ can thus be described as an integral $\mathcal{J}(r, t)$ over $\mathfrak{p} \times \mathfrak{p}^{(8)}$. We argue that it is equal to $Z_{\mathfrak{o}}^{[3]}(r, t)$, which may be computed from the integer sequence $a_{n}^{[3]}, n \in \mathbb{N}_{0}$, defined by

$$
\begin{aligned}
a_{n}^{[3]} & :=\#\left\{\mathbf{y}+\left(\mathfrak{p}^{n+1}\right)^{(8)} \mid \mathbf{y} \in \mathfrak{p}^{(8)} \text { such that }\left\|\left\{p y_{1}, p y_{2}, y_{3}, p^{n+1}\right\}\right\|_{\mathfrak{p}}=q^{-n-1}\right\} \\
& =\#\left\{\mathbf{y}+\left(\mathfrak{p}^{n+1}\right)^{(8)} \mid \mathbf{y} \in \mathfrak{p}^{(8)} \text { such that } y_{1}, y_{2} \in \mathfrak{p}^{n} \text { and } y_{3} \in \mathfrak{p}^{n+1}\right\} \\
& =\left|\mathfrak{p}^{\max \{1, n\}}: \mathfrak{p}^{n+1}\right|^{2} \cdot\left|\mathfrak{p}^{(5)}:\left(\mathfrak{p}^{n+1}\right)^{(5)}\right| \\
& = \begin{cases}1 & \text { if } n=0, \\
q^{5 n+2} & \text { if } n \geq 1,\end{cases}
\end{aligned}
$$

describing the lifting behaviour of points modulo $\mathfrak{p}^{n+1}$ on the variety defined by the integrand of $z_{\mathfrak{o}}^{[3]}(r, t)$. Indeed, we observe that the numbers $m_{l}^{[3]}$ defined in (5.11) satisfy

$$
m_{l}^{[3]}=q^{-8 l} a_{l-1}^{[3]}-q^{-8(l+1)} a_{l}^{[3]} .
$$

The following proposition shows that the integral $\mathcal{J}(r, t)$ over $\mathfrak{p} \times \mathfrak{p}^{(8)}$ is equal to $\mathcal{Z}_{\mathfrak{0}}^{[3]}(r, t)$.

Proposition 5.1. For $n \in \mathbb{N}_{0}$ the set

$$
\begin{aligned}
A_{n}^{[3]}:=\left\{\mathbf{a}+p^{n+1} \Lambda \in \Lambda / p^{n+1} \Lambda \mid \mathbf{a} \equiv\left(\begin{array}{lll}
0 & 1 & 0 \\
0 & 0 & 0 \\
0 & 0 & 0
\end{array}\right) \text { modulo } p \Lambda\right. \\
\\
\left.\quad \text { and }\left|\mathfrak{s l}_{3}(\mathfrak{o}): \mathrm{C}_{\mathfrak{s l}_{3}(\mathfrak{o})}\left(\mathbf{a}+p^{n+1} \Lambda\right)\right|=q^{4(n+1)}\right\}
\end{aligned}
$$

has cardinality $a_{n}^{[3]}$. 
Proof. The case $n=0$ is a simple computation. Indeed, the only candidate for an element of $A_{0}^{[3]}$ is $\mathbf{a}_{0}+p \Lambda$, where $\mathbf{a}_{0}:=\left(\begin{array}{lll}0 & 1 & 0 \\ 0 & 0 & 0 \\ 0 & 0 & 0\end{array}\right)$, and a short computation reveals that, indeed,

$$
\left|\mathfrak{s l}_{3}(\mathfrak{o}): \mathrm{C}_{\mathfrak{s l}_{3}(\mathfrak{o})}\left(\mathbf{a}_{0}+p \Lambda\right)\right|=\left|\mathfrak{s l}_{3}\left(\mathbb{F}_{q}\right): \mathrm{C}_{\mathfrak{s l}_{3}\left(\mathbb{F}_{q}\right)}\left(\overline{\mathbf{a}_{0}}\right)\right|=q^{4}
$$

where $\overline{\mathbf{a}_{0}}$ denotes the image of $\mathbf{a}_{0}$ in $\mathfrak{s l}_{3}\left(\mathbb{F}_{q}\right)$; cf. $(\overline{\text { B.2. }})$. Thus $\left|A_{0}^{[3]}\right|=1$, as claimed.

Now suppose that $n \geq 1$. Arguing by induction on $n$, we prove in fact a little more than stated in the proposition. For any $l \in \mathbb{N}$, let $T_{\mathfrak{o}}(l)$ denote representatives for $\mathfrak{o} / \mathfrak{p}^{l}$ derived from the Teichmüller representatives for $\mathfrak{o} / \mathfrak{p}$; see (5.1).

Claim. Every matrix $\mathbf{a} \in \Lambda$ with $\mathbf{a}+p^{n+1} \Lambda \in A_{n}^{[3]}$ can be conjugated by elements of $\mathrm{GL}_{3}^{1}(\mathfrak{o})$ to the 'normal' form

$$
\left(\begin{array}{ccc}
0 & 1 & 0 \\
2 p^{2} c^{2} & p c & 0 \\
0 & 0 & -p c
\end{array}\right)+p^{n}\left(\begin{array}{ccc}
0 & 0 & 0 \\
0 & 0 & y_{3} \\
y_{2} & 0 & 0
\end{array}\right) \quad \text { modulo } p^{n+1} \Lambda
$$

where $c \in T_{\mathfrak{o}}(n)$ and $y_{2}, y_{3} \in T_{\mathfrak{o}}(1)$. These matrices modulo $p^{n+1} \Lambda$ form a complete set of representatives for the $\mathrm{GL}_{3}^{1}(\mathfrak{o})$-orbits comprising $A_{n}^{[3]}$. Moreover, the index in $\mathrm{GL}_{3}^{1}(\mathfrak{o})$ of the centraliser of any such matrix modulo $p^{n+1} \Lambda$ is $q^{4 n}$, and thus $\left|A_{n}^{[3]}\right|=$ $\left|T_{\mathfrak{o}}(n)\right|\left|T_{\mathfrak{o}}(1)\right|^{2} q^{4 n}=q^{5 n+2}$, as wanted.

Finally, every matrix $\mathbf{a} \in \Lambda$ with $\mathbf{a}+p^{n+2} \Lambda \in A_{n+1}^{[3]}$ can be conjugated by elements of $\mathrm{GL}_{3}^{1}(\mathfrak{o})$ to a matrix which is, modulo $p^{n+1} \Lambda$, of the normal form above and satisfies the extra condition $y_{2}=y_{3}=0$.

As indicated we use induction on $n$. Let $c \in T_{\mathfrak{o}}(n-1)$ and put

$$
\mathbf{a}_{c}:=\left(\begin{array}{ccc}
0 & 1 & 0 \\
2 p^{2} c^{2} & p c & 0 \\
0 & 0 & -p c
\end{array}\right)
$$

The eigenvalues of $\mathbf{a}_{c}$ are $-p c$ and $2 p c$ with multiplicities 2 and 1 respectively. Hence $c$ is an invariant of the $\mathrm{GL}_{3}^{1}(\mathfrak{o})$-orbit of $\mathbf{a}_{c}$ modulo $\mathfrak{s t}_{3}^{n}(\mathfrak{o})$. In view of our discussion of the case $n=0$, if $n=1$, or the induction hypothesis, if $n>1$, it suffices to work out representatives of the elements of $A_{n}^{[3]}$ within the set $\mathbf{a}_{c}+\mathfrak{s l}_{3}^{n}(\mathfrak{o})$ modulo $\mathfrak{s l}_{3}^{n+1}(\mathfrak{o})$. We consider the set $\mathbf{a}_{c}+\mathfrak{s l}_{3}^{n}(\mathfrak{o})$ modulo $\mathfrak{s l}_{3}^{n+1}(\mathfrak{o})$, up to conjugation by $\mathrm{GL}_{3}^{n}(\mathfrak{o})$. Let

$$
\mathbf{x}:=\left(\begin{array}{ccc}
x_{1} & x_{2} & x_{3} \\
x_{4} & x_{5} & x_{6} \\
x_{7} & x_{8} & x_{9}
\end{array}\right) \in \operatorname{Mat}_{3}(\mathfrak{o})
$$

Then

$$
\begin{aligned}
{\left[\mathbf{a}_{c}, \mathbf{x}\right] } & =\left(\begin{array}{ccc}
x_{4}-2 p^{2} c^{2} x_{2} & \left(x_{5}-x_{1}\right)-p c x_{2} & x_{6}+p c x_{3} \\
p c\left(x_{4}+2 p c\left(x_{1}-x_{5}\right)\right) & -x_{4}+2 p^{2} c^{2} x_{2} & 2 p c\left(x_{6}+p c x_{3}\right) \\
-p c\left(x_{7}+2 p c x_{8}\right) & -x_{7}-2 p c x_{8} & 0
\end{array}\right) \\
& \equiv_{\mathfrak{p}}\left(\begin{array}{ccc}
x_{4} & x_{5}-x_{1} & x_{6} \\
0 & -x_{4} & 0 \\
0 & -x_{7} & 0
\end{array}\right) .
\end{aligned}
$$


If $\mathbf{b}=\mathbf{a}_{c}+p^{n} \mathbf{y} \in \mathbf{a}_{c}+\mathfrak{s l}_{3}^{n}(\mathfrak{o})$ and $g=1+p^{n} \mathbf{x} \in \mathrm{GL}_{3}^{n}(\mathfrak{o})$, then

$$
\begin{aligned}
g^{-1} \mathbf{b} g & \equiv\left(1-p^{n} \mathbf{x}\right)\left(\mathbf{a}_{c}+p^{n} \mathbf{y}\right)\left(1+p^{n} \mathbf{x}\right) \\
& \equiv \mathbf{a}_{c}+p^{n}\left(\mathbf{y}+\left[\mathbf{a}_{c}, \mathbf{x}\right]\right)
\end{aligned}
$$

modulo $\mathfrak{s l}_{3}^{n+1}(\mathfrak{o})$. In view of $(\underline{5.14})$ this shows that the elements

$$
\mathbf{b}_{c}\left(y_{1}, y_{2}, y_{3}, y_{4}\right):=\mathbf{a}_{c}+p^{n}\left(\begin{array}{ccc}
0 & 0 & 0 \\
y_{1} & y_{4} & y_{3} \\
y_{2} & 0 & -y_{4}
\end{array}\right)
$$

with $y_{1}, y_{2}, y_{3}, y_{4} \in T_{\mathfrak{o}}(1)$ form a complete set of representatives for the $\mathrm{GL}_{3}^{n}(\mathfrak{o})$-orbits of $\mathbf{a}_{c}+\mathfrak{s i n}_{3}^{n}(\mathfrak{o})$ modulo $\mathfrak{s l}_{3}^{n+1}(\mathfrak{o})$, and indeed modulo $p^{n+1} \Lambda$.

Consider one of these lifts, $\mathbf{b}=\mathbf{b}_{c}\left(y_{1}, y_{2}, y_{3}, y_{4}\right)$. In order to simplify the notation, it is convenient to use the fact that $\mathbf{b}=\mathbf{b}_{\tilde{c}}\left(y_{1}, y_{2}, y_{3}, 0\right)$, where $\tilde{c}:=c+p^{n-1} y_{4} \in T_{\mathfrak{o}}(n)$, and to work with

$$
\mathbf{a}_{\tilde{c}}:=\left(\begin{array}{ccc}
0 & 1 & 0 \\
2 p^{2} \tilde{c}^{2} & p \tilde{c} & 0 \\
0 & 0 & -p \tilde{c}
\end{array}\right)
$$

instead of $\mathbf{a}_{c}$. In order to describe the centraliser index of $\mathbf{b}+p^{n+1} \Lambda$ in $\mathfrak{s l}_{3}(\mathfrak{o})$, we consider again a generic matrix $\mathbf{x}$ as in (5.12), now with the additional restriction that $\mathbf{x} \in \mathfrak{s l}_{3}(\mathfrak{o})$, viz. $x_{1}+x_{5}+x_{9}=0$. One computes

$$
\begin{aligned}
& {[\mathbf{b}, \mathbf{x}]=\left[\mathbf{a}_{\tilde{c}}, \mathbf{x}\right]+} \\
& p^{n}\left(\begin{array}{ccc}
-y_{1} x_{2}-y_{2} x_{3} & 0 & -y_{3} x_{2} \\
y_{1}\left(x_{5}-x_{1}\right)-y_{2} x_{6}+y_{3} x_{7} & y_{1} x_{2}+y_{3} x_{8} & y_{1} x_{3}+y_{3}\left(x_{9}-x_{5}\right) \\
y_{2}\left(x_{1}-x_{9}\right)-y_{1} x_{8} & y_{2} x_{2} & y_{2} x_{3}-y_{3} x_{8}
\end{array}\right) .
\end{aligned}
$$

Taking into account (5.13), the condition $[\mathbf{b}, \mathbf{x}] \equiv 0$ modulo $p^{n+1} \Lambda$ can be expressed in terms of the following list of restrictions on the entries of $\mathbf{x}$, involving the parameters $y_{1}, y_{2}, y_{3} \in T_{\mathfrak{o}}(1)$ :

(i) $x_{4}-2 p^{2} \tilde{c}^{2} x_{2}-p^{n}\left(y_{1} x_{2}+y_{2} x_{3}\right) \equiv_{p^{n+1}}-x_{4}+2 p^{2} \tilde{c}^{2} x_{2}+p^{n}\left(y_{1} x_{2}+y_{3} x_{8}\right)$ from the $(1,1)$ - and (2,2)-entries, equivalently $2 x_{4} \equiv_{p^{n+1}} 4 p^{2} \tilde{c}^{2} x_{2}+p^{n}\left(2 y_{1} x_{2}+y_{2} x_{3}+y_{3} x_{8}\right)$,

(ii) $x_{5} \equiv_{p^{n+1}} x_{1}+p \tilde{c} x_{2}$ from the $(1,2)$-entry,

(iii) $x_{6} \equiv_{p^{n+1}}-p \tilde{c} x_{3}+p^{n} y_{3} x_{2}$ from the $(1,3)$-entry,

(iv) $x_{7} \equiv_{p^{n+1}}-2 p \tilde{c} x_{8}+p^{n} y_{2} x_{2}$ from the $(3,2)$-entry,

(v) $0 \equiv_{p}-y_{2} x_{6}+y_{3} x_{7}$ from the $(2,1)$-entry, but this condition becomes redundant if $x_{6}, x_{7} \equiv_{p} 0$,

(vi) $0 \equiv_{p} y_{2}\left(x_{1}-x_{9}\right)-y_{1} x_{8} \equiv_{p} y_{2}\left(x_{1}+x_{5}+x_{9}\right)-y_{1} x_{8} \equiv_{p}-y_{1} x_{8}$ from the $(3,1)$-entry and (ii),

(vii) $0 \equiv_{p} y_{1} x_{3}+y_{3}\left(x_{9}-x_{5}\right) \equiv_{p} y_{1} x_{3}+y_{3}\left(x_{1}+x_{5}+x_{9}\right) \equiv_{p} y_{1} x_{3}$ from the $(2,3)$-entry and (ii),

(viii) $-x_{4}+2 p^{2} \tilde{c}^{2} x_{2}+p^{n}\left(y_{1} x_{2}+y_{3} x_{8}\right) \equiv_{p^{n+1}} p^{n}\left(y_{2} x_{3}-y_{3} x_{8}\right)$ from the $(2,2)$ - and (3,3)-entries, equivalently $x_{4} \equiv_{p^{n+1}} 2 p^{2} \tilde{c}^{2} x_{2}+p^{n}\left(y_{1} x_{2}-y_{2} x_{3}+2 y_{3} x_{8}\right)$.

If these conditions are to hold for $\mathbf{x} \in \mathfrak{s l}_{3}(\mathfrak{o})$, then the congruences (i)-(iv) show that the entries $x_{4}, x_{5}, x_{6}, x_{7}$ are determined completely modulo $p^{n+1}$ by the remaining entries $x_{1}, x_{2}, x_{3}, x_{8}, x_{9}$. Because $x_{1}+x_{5}+x_{9}=\operatorname{Tr}(\mathbf{x})=0$, we can also think of $x_{9}$ as being determined by $x_{1}$. We observe that $\left|\mathfrak{s l}_{3}(\mathfrak{o}): \mathrm{C}_{\mathfrak{s l}_{3}(\mathfrak{o})}\left(\mathbf{b}+p^{n+1} \Lambda\right)\right|=q^{4(n+1)}$ (and not 
larger) if and only if one can choose $x_{1}, x_{2}, x_{3}, x_{8}$ freely, i.e. if the remaining conditions (v)-(viii) do not impose extra restrictions.

In fact, the congruence (v) will be automatically satisfied, as indicated, because $x_{6}, x_{7} \equiv_{p} 0$. As $x_{1}+x_{5}+x_{9}=\operatorname{Tr}(\mathbf{x})=0$, the conditions (vi) and (vii) will give rise to restrictions on $x_{8}$ or $x_{3}$, unless $y_{1} \equiv_{p} 0$. Finally, the last condition (viii) is equivalent to the condition (i), since $p=3$.

The discussion so far shows that, with respect to the action of $\mathrm{GL}_{3}^{n}(\mathfrak{o})$, the intersection of $A_{n}^{[3]}$ and $\left(\mathbf{a}_{c}+\mathfrak{s l}_{3}^{n}(\mathfrak{o})\right) / p^{n+1} \Lambda$ consists of $q^{3}$ orbits, represented by matrices

$$
\mathbf{b}_{c}\left(0, y_{2}, y_{3}, y_{4}\right)
$$

and each of size $q^{4}$. By induction, there are $q^{5(n-1)}$ matrices modulo $p^{n-1} \Lambda$, represented by matrices such as $\mathbf{a}_{c}$, which lift to elements of $A_{n}^{[3]}$. Hence $\left|A_{n}^{[3]}\right|=q^{5(n-1)} q^{3+4}=q^{5 n+2}$. In order to show that the $\left|T_{\mathfrak{o}}(n-1)\right| q^{3}=q^{n+2}$ matrices $\mathbf{b}_{c}\left(0, y_{2}, y_{3}, y_{4}\right)$ form a complete set of representatives for the $\mathrm{GL}_{3}^{1}(\mathfrak{o})$-orbits comprising $A_{n}^{[3]}$, it suffices to show that for any one of them, $\mathbf{b}=\mathbf{b}_{c}\left(0, y_{2}, y_{3}, y_{4}\right)$ say, one has

$$
\left|\mathrm{GL}_{3}^{1}(\mathfrak{o}): \mathrm{C}_{\mathrm{GL}_{3}^{1}(\mathfrak{o})}\left(\mathbf{b}+p^{n+1} \Lambda\right)\right|=q^{4 n} .
$$

We make three observations. Firstly, a straightforward translation between $\mathrm{GL}_{3}^{1}(\mathfrak{o})$ and its Lie lattice $\mathfrak{g l}_{3}^{1}(\mathfrak{o})$ yields

$$
\left|\mathrm{GL}_{3}^{1}(\mathfrak{o}): \mathrm{C}_{\mathrm{GL}_{3}^{1}(\mathfrak{o})}\left(\mathbf{b}+p^{n+1} \Lambda\right)\right|=\left|\mathfrak{g l}_{3}^{1}(\mathfrak{o}): \mathrm{C}_{\mathfrak{g l}_{3}^{1}(\mathfrak{o})}\left(\mathbf{b}+p^{n+1} \Lambda\right)\right| .
$$

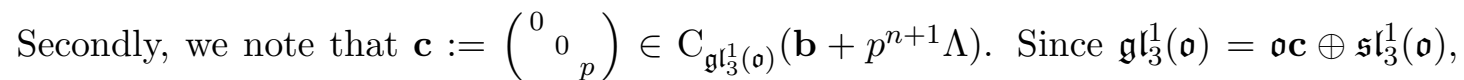
this implies that $\mathfrak{g l}_{3}^{1}(\mathfrak{o})=\mathrm{C}_{\mathfrak{g l}_{3}^{1}(\mathfrak{o})}\left(\mathbf{b}+p^{n+1} \Lambda\right)+\mathfrak{s l}_{3}^{1}(\mathfrak{o})$, and consequently

$$
\left|\mathfrak{g l}_{3}^{1}(\mathfrak{o}): \mathrm{C}_{\mathfrak{g l}_{3}^{1}(\mathfrak{o})}\left(\mathbf{b}+p^{n+1} \Lambda\right)\right|=\left|\mathfrak{s l}_{3}^{1}(\mathfrak{o}): \mathrm{C}_{\mathfrak{s l}}(\mathfrak{o})\left(\mathbf{b}+p^{n+1} \Lambda\right)\right|
$$

Finally, we observe that the property $\left|\mathfrak{s l}_{3}(\mathfrak{o}): \mathrm{C}_{\mathfrak{s l}_{3}(\mathfrak{o})}\left(\mathbf{b}+p^{n+1} \Lambda\right)\right|=q^{4(n+1)}$ is, in fact, equivalent to $\left|\mathfrak{s l}_{3}(\mathfrak{o}): \mathrm{C}_{\mathfrak{s l}_{3}(\mathfrak{o})}(\mathbf{b}+p \Lambda)\right|=q^{4}$ so that

$$
\left|\mathfrak{s} \mathfrak{l}_{3}^{1}(\mathfrak{o}): \mathrm{C}_{\mathfrak{s} l_{3}^{1}(\mathfrak{o})}\left(\mathbf{b}+p^{n+1} \Lambda\right)\right|=q^{-4} q^{4(n+1)}=q^{4 n} .
$$

These three observations yield (5.16), as wanted.

To establish the last part of the induction claim, consider the relevance of the values of $y_{2}, y_{3}$ for lifting one of these matrices one step further. Consider $\mathbf{b}=\mathbf{b}_{c}\left(y_{1}, y_{2}, y_{3}, y_{4}\right)=$ $\mathbf{b}_{\tilde{c}}\left(y_{1}, y_{2}, y_{3}, 0\right)$, where $\tilde{c}:=c+p^{n-1} y_{4} \in T_{\mathfrak{o}}(n+1)$, similarly as above, but allowing $y_{1}, \ldots, y_{4} \in T_{\mathfrak{o}}(2)$. Then the centraliser condition $[\mathbf{b}, \mathbf{x}] \equiv 0$ modulo $p^{n+2} \Lambda$ leads, on the diagonal entries (cf. conditions (i) and (viii) in the list above), to the restriction

$$
\begin{aligned}
0 & \equiv{ }_{p^{n+2}}\left(4 p^{2} \tilde{c}^{2} x_{2}+p^{n}\left(2 y_{1} x_{2}+y_{2} x_{3}+y_{3} x_{8}\right)\right)-2 \cdot\left(p^{2} \tilde{c}^{2} x_{2}-p^{n}\left(y_{1} x_{2}-y_{2} x_{3}+2 y_{3} x_{8}\right)\right) \\
& =p^{n}\left(3 y_{2} x_{3}-3 y_{3} x_{8}\right)
\end{aligned}
$$

which is equivalent to $0 \equiv_{p} y_{2} x_{3}-y_{3} x_{8}$, as $p=3$. Unless both $y_{2}$ and $y_{3}$ are congruent to 0 modulo $p$, this leads to an unwanted restriction on $x_{3}$ and $x_{8}$. This shows that it is enough to look for representatives of the elements of $A_{n+1}^{[3]}$ within the sets $\mathbf{a}_{\tilde{c}}+\mathfrak{s l}_{3}^{n+1}(\mathfrak{o})$ modulo $\mathfrak{s}_{3}^{n+2}(\mathfrak{o})$, where $\mathbf{a}_{\tilde{c}}$ arises from $\tilde{c} \equiv c$ modulo $p^{n-1}$. 
5.2.2. Next we consider elements belonging to cosets modulo $p \Lambda$ of type B. Similarly as for type $\mathrm{C}$, we claim that the relevant integral for type $\mathrm{B}$ is equal to the $z_{\mathfrak{o}}^{[2]}(r, t)$. The latter may be computed easily from the integer sequence $a_{n}^{[2]}, n \in \mathbb{N}_{0}$, defined by

$$
a_{n}^{[2]}:=\#\left\{\mathbf{y}+\left(\mathfrak{p}^{n+1}\right)^{(8)} \mid \mathbf{y} \in \mathfrak{p}^{(8)} \text { such that }|p|_{\mathfrak{p}}=q^{-n-1}\right\}= \begin{cases}1 & \text { if } n=0, \\ 0 & \text { if } n \geq 1,\end{cases}
$$

observing that the numbers $m_{l}^{[2]}$ defined in (5.10) satisfy

$$
m_{l}^{[2]}=q^{-8 l} a_{l-1}^{[2]}-q^{-8(l+1)} a_{l}^{[2]} .
$$

The claim follows from the following proposition.

Proposition 5.2. For $n \in \mathbb{N}_{0}$ the set

$$
\begin{aligned}
A_{n}^{[2]}:=\left\{\mathbf{a}+p^{n+1} \Lambda \in \Lambda / p^{n+1} \Lambda \mid \mathbf{a} \equiv\left(\begin{array}{lll}
0 & 1 & 0 \\
0 & 0 & 1 \\
0 & 0 & 0
\end{array}\right) \text { modulo } p \Lambda\right. \\
\\
\left.\quad \text { and }\left|\mathfrak{s l}_{3}(\mathfrak{o}): \mathrm{C}_{\mathfrak{s l}_{3}(\mathfrak{o})}\left(\mathbf{a}+p^{n+1} \Lambda\right)\right|=q^{4(n+1)}\right\}
\end{aligned}
$$

has cardinality $a_{n}^{[2]}$.

Proof. The case $n=0$ is a simple computation. The only candidate for an element of $A_{0}^{[2]}$ is $\mathbf{a}_{0}+p \Lambda$, where $\mathbf{a}_{0}:=\left(\begin{array}{lll}0 & 1 & 0 \\ 0 & 0 & 1 \\ 0 & 0 & 0\end{array}\right)$, and a short computation reveals that

$$
\left|\mathfrak{s l}_{3}(\mathfrak{o}): \mathrm{C}_{\mathfrak{s l}(\mathfrak{o})}\left(\mathbf{a}_{0}+p \Lambda\right)\right|=q^{4} .
$$

Indeed, for

$$
\mathbf{x}=\left(\begin{array}{lll}
x_{1} & x_{2} & x_{3} \\
x_{4} & x_{5} & x_{6} \\
x_{7} & x_{8} & x_{9}
\end{array}\right) \in \mathfrak{s l}_{3}(\mathfrak{o})
$$

the commutator identity

$$
\left[\mathbf{a}_{0}, \mathbf{x}\right]=\left(\begin{array}{ccc}
x_{4} & x_{5}-x_{1} & x_{6}-x_{2} \\
x_{7} & x_{8}-x_{4} & x_{9}-x_{5} \\
0 & -x_{7} & -x_{8}
\end{array}\right)
$$

shows that $\mathbf{x} \in \mathrm{C}_{\mathfrak{s l}_{3}(\mathfrak{o})}\left(\mathbf{a}_{0}+p \Lambda\right)$ if and only if the following congruences are satisfied:

$$
x_{4} \equiv_{p}-x_{8}, \quad x_{5} \equiv_{p} x_{9} \equiv_{p} x_{1}, \quad x_{6} \equiv_{p} x_{2}, \quad x_{7} \equiv_{p} 0,
$$

where $x_{1}, x_{2}, x_{3}, x_{8}$ can be chosen freely. Thus $\left|A_{0}^{[2]}\right|=1$, as claimed.

Next suppose that $n \geq 1$, and consider a lift $\mathbf{b} \in \mathbf{a}_{0}+\mathfrak{s l}_{3}^{1}(\mathfrak{o})$ modulo $\mathfrak{s l}_{3}^{2}(\mathfrak{o})$. Replacing $\mathbf{b}$ by a conjugate under $\mathrm{GL}_{3}^{1}(\mathfrak{o})$ if necessary, we may assume, by a computation similar to (5.15), that $\mathbf{b}$ is of the form

$$
\mathbf{b}=\mathfrak{b}\left(y_{1}, y_{2}\right)=\mathbf{a}_{0}+\left(\begin{array}{ccc}
0 & 0 & 0 \\
0 & 0 & 0 \\
p y_{1} & p y_{2} & 0
\end{array}\right)=\left(\begin{array}{ccc}
0 & 1 & 0 \\
0 & 0 & 1 \\
p y_{1} & p y_{2} & 0
\end{array}\right)
$$

where $y_{1}, y_{2} \in T_{\mathfrak{o}}(1)$ are Teichmüller representatives in $\mathfrak{o}$. Suppose that $\mathbf{x}$, as in (5.17), lies in $\mathrm{C}_{\mathfrak{s} \mathfrak{l}_{3}(\mathfrak{o})}\left(\mathbf{b}+p^{2} \Lambda\right)$. Then the commutator identity

$$
[\mathbf{b}, \mathbf{x}]=\left[\mathbf{a}_{0}, \mathbf{x}\right]+\left(\begin{array}{ccc}
-p y_{1} x_{3} & -p y_{2} x_{3} & 0 \\
-p y_{1} x_{6} & -p y_{2} x_{6} & 0 \\
p\left(y_{1}\left(x_{1}-x_{9}\right)+y_{2} x_{4}\right) & p\left(y_{1} x_{2}+y_{2}\left(x_{5}-x_{9}\right)\right) & p\left(y_{1} x_{3}+y_{2} x_{6}\right)
\end{array}\right)
$$


in conjunction with (5.18), reveals that

$$
x_{4}-p y_{1} x_{3} \equiv_{p^{2}}\left(x_{8}-x_{4}\right)-p y_{2} x_{6} \equiv_{p^{2}}-x_{8}+p\left(y_{1} x_{3}+y_{2} x_{6}\right),
$$

hence $3 x_{4} \equiv_{p^{2}} 3 x_{8} \equiv_{p^{2}} 0$ irrespective of the particular values of $y_{1}, y_{2}$. Furthermore, inspection of the $(1,2)$ - and $(2,3)$-entries of the commutator identity shows that $x_{1} \equiv_{p}$ $x_{5} \equiv_{p^{2}} x_{9}$. Since $x_{1}+x_{5}+x_{9} \equiv_{p} 0$, this yields $x_{1} \equiv_{p} x_{5} \equiv_{p} x_{9} \equiv_{p} 0$. As before, $x_{4}, x_{5}, x_{6}, x_{7}, x_{9}$ are determined by the values of $x_{1}, x_{2}, x_{3}, x_{8}$, but the latter satisfy the extra condition $x_{1} \equiv_{p} x_{8} \equiv_{p} 0$. Hence the relevant index $\left|\mathfrak{s l}_{3}(\mathfrak{o}): \mathrm{C}_{\mathfrak{s l}_{3}(\mathfrak{o})}\left(\mathbf{b}+p^{2} \Lambda\right)\right| \geq q^{10}$. This shows that $A_{1}^{[2]}=\varnothing$ and consequently $A_{n}^{[2]}=\varnothing$ for all $n \geq 1$.

5.3. In order to conclude the justification of the equations (5.4) we explain how one obtains the summand $\mathcal{S}_{2}(r, t)$. For this we decompose $\Lambda \backslash \mathfrak{s l}_{3}(\mathfrak{o})$ into cosets modulo $p \Lambda$. As $p=3$, every element in this domain is of the form $u\left(p^{-1} \mathrm{Id}+\mathbf{x}\right)$, where $u \in \mathfrak{o}^{*}$ and $\mathbf{x} \in$ $\mathfrak{g l}_{3}(\mathfrak{o})$ has trace -1 . We are thus led to decomposing the finite affine space of matrices $\left\{\mathbf{x} \in \mathfrak{g l}_{3}\left(\mathbb{F}_{q}\right) \mid \operatorname{Tr}(\mathbf{x})=-1\right\}$ into cosets modulo the 1-dimensional subspace $\mathbb{F}_{q} \overline{\mathbf{z}}$, spanned by the reduction modulo $\mathfrak{p}$ of $\mathbf{z}:=\mathbf{h}_{12}-\mathbf{h}_{23}$. Of course, the latter coincides with the space of scalar matrices over $\mathbb{F}_{q}$. An overview of the orbits in $\left\{\mathbf{x} \in \mathfrak{g l}_{3}\left(\mathbb{F}_{q}\right) \mid \operatorname{Tr}(\mathbf{x})=-1\right\}$ under the adjoint action of $\mathrm{GL}_{3}\left(\mathbb{F}_{q}\right)$ is provided in Table 5.2 , see Appendix $\mathbb{C}$ for a short discussion. The corresponding total number of cosets modulo $\mathbb{F} q \overline{\mathbf{z}}$, given in the last column of the table, is obtained upon division by $q$. Indeed, a short calculation confirms that the sizes of the orbits listed in Table 5.2 add up to $q^{7}$ so that with $q-1$ choices for $u$ modulo $\mathfrak{p}$ we obtain $(q-1) q^{7}=|\Lambda: p \Lambda|-\left|\mathfrak{s l}_{3}(\mathfrak{o}): p \Lambda\right|$, as wanted.

\begin{tabular}{|c|l|l|l|l|}
\hline type & & no. of orbits & size of each orbit & total number modulo $\mathbb{F}_{q} \overline{\mathbf{z}}$ \\
\hline \hline $\mathrm{G}$ & irregular & $q$ & $\left(q^{2}+q+1\right) q^{2}$ & $\left(q^{2}+q+1\right) q^{2}$ \\
$\mathrm{H}$ & regular & $(q-3) q / 6$ & $\left(q^{2}+q+1\right)(q+1) q^{3}$ & $(q-3)\left(q^{2}+q+1\right)(q+1) q^{3} / 6$ \\
$\mathrm{I}$ & regular & $(q-1) q / 2$ & $\left(q^{3}-1\right) q^{3}$ & $\left(q^{3}-1\right)(q-1) q^{3} / 2$ \\
$\mathrm{~J}$ & regular & $q^{2} / 3$ & $\left(q^{2}-1\right)(q-1) q^{3}$ & $\left(q^{2}-1\right)(q-1) q^{4} / 3$ \\
$\mathrm{~K}$ & regular & $q$ & $\left(q^{3}-1\right)(q+1) q^{2}$ & $\left(q^{3}-1\right)(q+1) q^{2}$ \\
\hline
\end{tabular}

TABLE 5.2. Adjoint orbits in $\left\{\mathbf{x} \in \mathfrak{g l}_{3}\left(\mathbb{F}_{q}\right) \mid \operatorname{Tr}(\mathbf{x})=-1\right\}$ under the action of $\mathrm{GL}_{3}\left(\mathbb{F}_{q}\right), q \equiv_{3} 0$

That $z_{\mathfrak{o}}^{[0]}(r, t)$ is the correct integral for the types $\mathrm{H}, \mathrm{I}, \mathrm{J}, \mathrm{K}$ is clear, because they correspond to regular elements modulo $p$. We need to link the contributions by irregular elements belonging to cosets of type $\mathrm{G}$ to the integral $z_{\mathfrak{o}}^{[1]}(r, t)$, as shown within the summand $\mathcal{S}_{2}(r, t)$ in (5.4).

Hence let us consider elements belonging to cosets modulo $p \Lambda$ of type G. A typical coset of this type is $\mathbf{a}_{0}+p \Lambda$, where $\mathbf{a}_{0}:=\left(\begin{array}{ccc}0 & 0 & 0 \\ 0 & 0 & 0 \\ 0 & 0 & -1\end{array}\right)$, and each coset has measure $\mu(p \Lambda)=$ $q^{-7}$. The determinant of the Jacobi matrix associated to $\iota_{0}: \Lambda \rightarrow \operatorname{Hom}_{\mathfrak{o}}\left(\mathfrak{s l}_{3}(\mathfrak{o}), \mathfrak{o}\right)$ contributes another factor $|3|_{\mathfrak{p}}=q^{-1}$. The integral over $\mathfrak{p} \times(\mathbf{a}+p \Lambda)$ with Jacobi factor $q^{-1}$ can thus be described as an integral over $\mathfrak{p} \times \mathfrak{p}^{(8)}$. Similarly as in cases B and C, we claim that this integral equals $z_{\mathfrak{o}}^{[1]}(r, t)$. The latter may be computed from the integer 
sequence $a_{n}^{[1]}, n \in \mathbb{N}_{0}$, defined by

$$
\begin{aligned}
a_{n}^{[1]} & :=\#\left\{\mathbf{y}+\left(\mathfrak{p}^{n+1}\right)^{(8)} \mid \mathbf{y} \in \mathfrak{p}^{(8)} \text { such that }\left\|\left\{y_{1}, y_{2}, y_{3}, p^{n+1}\right\}\right\|_{\mathfrak{p}}=q^{-n-1}\right\} \\
& =\#\left\{\mathbf{y}+\left(\mathfrak{p}^{n+1}\right)^{(8)} \mid \mathbf{y} \in \mathfrak{p}^{(8)} \text { such that } y_{1}, y_{2}, y_{3} \in \mathfrak{p}^{n+1}\right\} \\
& =\left|\mathfrak{p}^{(5)} /\left(\mathfrak{p}^{n+1}\right)^{(5)}\right| \\
& =q^{5 n}
\end{aligned}
$$

describing the lifting behaviour of points modulo $\mathfrak{p}^{n+1}$ on the variety defined by the integrand of $z_{\mathfrak{o}}^{[1]}(r, t)$. Indeed, we observe that the numbers $m_{l}^{[1]}$ defined in (5.7) satisfy

$$
m_{l}^{[1]}=q^{-8 l} a_{l-1}^{[1]}-q^{-8(l+1)} a_{l}^{[1]} .
$$

The following proposition establishes the claim and thereby concludes the overall proof of Theorem 1.4.

Proposition 5.3. For $n \in \mathbb{N}_{0}$ the set

$$
\begin{aligned}
A_{n}^{[1]}:=\left\{\mathbf{a}+p^{n+1} \Lambda \in \Lambda / p^{n+1} \Lambda \mid \mathbf{a} \equiv p^{-1} \operatorname{Id}+\left(\begin{array}{ccc}
0 & 0 & 0 \\
0 & 0 & 0 \\
0 & 0 & -1
\end{array}\right) \text { modulo } p \Lambda\right. \\
\\
\left.\quad \text { and }\left|\mathfrak{s l}_{3}(\mathfrak{o}): \mathrm{C}_{\mathfrak{s l}_{3}(\mathfrak{o})}\left(\mathbf{a}+p^{n+1} \Lambda\right)\right|=q^{4(n+1)}\right\}
\end{aligned}
$$

has cardinality $a_{n}^{[1]}$.

Proof. We argue by induction on $n$. The case $n=0$ is a simple computation. Indeed, the only candidate for an element of $A_{0}^{[1]}$ is $\mathbf{a}_{0}+p \Lambda$, where $\mathbf{a}_{0}:=p^{-1} \operatorname{Id}+\left(\begin{array}{ccc}0 & 0 & 0 \\ 0 & 0 & 0 \\ 0 & 0 & -1\end{array}\right)$, and a short computation reveals that, indeed,

$$
\left|\mathfrak{s l}_{3}(\mathfrak{o}): \mathrm{C}_{\mathfrak{s l}_{3}(\mathfrak{o})}\left(\mathbf{a}_{0}+p \Lambda\right)\right|=\left|\mathfrak{s l}_{3}\left(\mathbb{F}_{q}\right): \mathrm{C}_{\mathfrak{s l}_{3}\left(\mathbb{F}_{q}\right)}\left(\overline{\mathbf{a}_{0}}\right)\right|=q^{4},
$$

where $\overline{\mathbf{a}_{0}}$ denotes the image of $\mathbf{a}_{0}$ in $\mathfrak{s l}_{3}\left(\mathbb{F}_{q}\right)$; cf. $(\overline{B .3})$. Thus $\left|A_{0}^{[1]}\right|=1$, as claimed.

Now suppose that $n \geq 1$. In fact, we will prove more than stated in the proposition. For any $l \in \mathbb{N}$, let $T_{\mathfrak{o}}(l)$ denote the representatives for $\mathfrak{o} / \mathfrak{p}^{l}$ derived from the Teichmüller representatives for $\mathfrak{o} / \mathfrak{p}$; see (5.1). By induction on $n$, the following assertions are proved below.

Claim. Every matrix $\mathbf{a} \in \Lambda$ with $\mathbf{a}+p^{n+1} \Lambda \in A_{n}^{[1]}$ can be conjugated by elements of $\mathrm{GL}_{3}^{1}(\mathfrak{o})$ to the 'normal' form

$$
p^{-1} \mathrm{Id}+\left(\begin{array}{ccc}
c & 0 & 0 \\
0 & c & 0 \\
0 & 0 & -1-2 c
\end{array}\right) \quad \text { modulo } p^{n+1} \Lambda,
$$

where $c \in T_{\mathfrak{o}}(n)$. These matrices modulo $p^{n+1} \Lambda$ form a complete set of representatives for the $\mathrm{GL}_{3}^{1}(\mathfrak{o})$-orbits comprising $A_{n}^{[1]}$. Moreover, the index in $\mathrm{GL}_{3}^{1}(\mathfrak{o})$ of the centraliser of any such matrix modulo $p^{n+1} \Lambda$ is $q^{4 n}$, and $\left|A_{n}^{[1]}\right|=\left|T_{\mathfrak{o}}(n)\right| q^{4 n}=q^{5 n}$, as wanted.

Let $c \in T_{\mathfrak{o}}(n)$ and put

$$
\mathbf{a}:=\mathbf{a}_{c}:=\left(\begin{array}{ccc}
c & 0 & 0 \\
0 & c & 0 \\
0 & 0 & -1-2 c
\end{array}\right) .
$$

Clearly, the eigenvalues of a are $c$ and $-1-2 c$ with multiplicities 2 and 1 respectively. Hence $c$ modulo $p^{n-1}$ is an invariant of the $\mathrm{GL}_{3}^{1}(\mathfrak{o})$-orbit of a modulo $p^{n} \Lambda$. By induction, 
it suffices to look for representatives of the elements of $A_{n}^{[1]}$ within the set $\mathbf{a}+\mathfrak{s l}_{3}^{n}(\mathfrak{o})$ modulo $p^{n+1} \Lambda$. We consider the set $\mathbf{a}+\mathfrak{s l}_{3}^{n}(\mathfrak{o})$ modulo $\mathfrak{s l}_{3}^{n+1}(\mathfrak{o})$, up to conjugation by $\mathrm{GL}_{3}^{n}(\mathfrak{o})$. Let

$$
\mathbf{x}:=\left(\begin{array}{cc}
A & \underline{b}^{\mathrm{t}} \\
\underline{d} & a
\end{array}\right) \in \operatorname{Mat}_{3}(\mathfrak{o}), \quad \text { where } A \in \operatorname{Mat}_{2}(\mathfrak{o}) \text { and } \underline{b}, \underline{d} \in \mathfrak{o}^{(2)}, a \in \mathfrak{o} .
$$

Then, as $p=3$,

$$
\begin{aligned}
{[\mathbf{a}, \mathbf{x}] } & =\left(\begin{array}{cc}
0 & (1+3 c) \underline{b}^{\mathrm{t}} \\
-(1+3 c) \underline{d} & 0
\end{array}\right) \\
& \equiv_{\mathfrak{p}}\left(\begin{array}{cc}
0 & \underline{b}^{\mathrm{t}} \\
\underline{d} & 0
\end{array}\right) .
\end{aligned}
$$

As in the proof of Proposition 5.1, the congruence (5.20) shows that the elements

$$
\mathbf{b}_{c}(Y):=\mathbf{a}+p^{n}\left(\begin{array}{cc}
Y & 0 \\
0 & -\operatorname{Tr}(Y)
\end{array}\right), \quad \text { with } Y=\left(\begin{array}{ll}
y_{1} & y_{2} \\
y_{3} & y_{4}
\end{array}\right) \in \operatorname{Mat}_{2}\left(T_{\mathfrak{o}}(1)\right),
$$

form a complete set of representatives for the $\mathrm{GL}_{3}^{n}(\mathfrak{o})$-orbits of $\mathbf{a}+\mathfrak{s l}_{3}^{n}(\mathfrak{o})$ modulo $\mathfrak{s l}_{3}^{n+1}(\mathfrak{o})$, and with the extra restriction $\operatorname{Tr}(Y)=0$ a complete set of representatives modulo $p^{n+1} \Lambda$.

Consider one of these lifts, $\mathbf{b}=\mathbf{b}_{c}(Y)$ and put $z:=\operatorname{Tr}(Y)$. In order to describe the centraliser index of $\mathbf{b}+p^{n+1} \Lambda$ in $\mathfrak{s l}_{3}(\mathfrak{o})$, we consider a generic matrix

$$
\mathbf{x}:=\left(\begin{array}{cc}
A & \underline{b}^{\mathrm{t}} \\
\underline{d} & -\operatorname{Tr}(A)
\end{array}\right) \in \mathfrak{s l}_{3}(\mathfrak{o}), \quad \text { where } A \in \operatorname{Mat}_{2}(\mathfrak{o}) \text { and } \underline{b}, \underline{d} \in \mathfrak{o}^{(2)}
$$

and compute

$$
[\mathbf{b}, \mathbf{x}]=[\mathbf{a}, \mathbf{x}]+p^{n}\left(\begin{array}{cc}
Y A-A Y & Y \underline{b}^{\mathrm{t}}-z \underline{b}^{\mathrm{t}} \\
z \underline{d}-\underline{d} Y & 0
\end{array}\right) .
$$

Taking into account (5.19), the condition $[\mathbf{b}, \mathbf{x}] \equiv 0$ modulo $p^{n+1} \Lambda$ can be expressed in terms of the following list of restrictions on the entries of $\mathbf{x}$, involving as parameters the matrix $Y \in \operatorname{Mat}_{2}\left(T_{\mathfrak{o}}(1)\right)$ and $z=\operatorname{Tr}(Y)$ :

(i) $Y A-A Y \equiv_{p} 0$,

(ii) $\left(\left(1+3 c-p^{n} z\right) \operatorname{Id}+p^{n} Y\right) \underline{b}^{\mathrm{t}} \equiv_{p^{n+1}} 0$,

(iii) $\underline{d}\left(\left(1+3 c-p^{n} z\right) \operatorname{Id}+p^{n} Y\right) \equiv_{p^{n+1}} 0$.

If these conditions are to hold then the last two congruences show that $\underline{b}$ and $\underline{d}$ are to be 0 modulo $p^{n+1}$. From this we observe that $\left|\mathfrak{s l}_{3}(\mathfrak{o}): \mathrm{C}_{\mathfrak{s l}_{3}(\mathfrak{o})}\left(\mathbf{a}+p^{n+1} \Lambda\right)\right|=q^{4(n+1)}$ (and not larger) if and only if one can choose $A$ freely, i.e. if the first condition does not impose extra restrictions. This implies that $Y$ is scalar, and $\operatorname{Tr}(Y)=0$ implies that $Y=0$. The proof concludes in analogy to the proof of Proposition 5.1

\section{Appendix A. Adjoint ACtion of $\mathrm{GL}_{2}\left(\mathbb{F}_{q}\right)$ ON $\mathfrak{s l}_{2}\left(\mathbb{F}_{q}\right)$}

In Section 3.3. we require an overview of the elements in $\mathfrak{s l}_{2}\left(\mathbb{F}_{q}\right)$ up to conjugacy under the group $\mathrm{GL}_{2}\left(\mathbb{F}_{q}\right)$. We distinguish four different types, labelled $0,1,2 \mathrm{a}, 2 \mathrm{~b}$. The total number of elements of each type and the isomorphism types of their centralisers in $\mathrm{SL}_{2}\left(\mathbb{F}_{q}\right)$ are summarised in Tables 3.1 and 3.2. We briefly discuss the four different types.

Type 0 consists of the zero matrix, which does not feature in our calculation but is shown for completeness. Its centraliser is the entire group $\mathrm{SL}_{2}\left(\mathbb{F}_{q}\right)$. 
Type 1 consists of nilpotent matrices with minimal polynomial equal to $X^{2}$ over $\mathbb{F}_{q}$. The centraliser of a typical element is

$$
\mathrm{C}_{\mathrm{SL}_{2}\left(\mathbb{F}_{q}\right)}\left(\left(\begin{array}{ll}
0 & 1 \\
0 & 0
\end{array}\right)\right)=\left\{\left(\begin{array}{ll}
a & b \\
0 & a
\end{array}\right) \mid a, b \in \mathbb{F}_{q}, a^{2}=1\right\}
$$

and matrices of type 1 are regular.

Type $2 \mathrm{a}$ consists of semisimple matrices with distinct eigenvalues $\lambda,-\lambda \in \mathbb{F}_{q} \backslash\{0\}$. The minimal polynomial of such elements over $\mathbb{F}_{q}$ is equal to $X^{2}-\lambda^{2}$. The centraliser of a typical element is

$$
\mathrm{C}_{\mathrm{SL}_{2}\left(\mathbb{F}_{q}\right)}\left(\left(\begin{array}{cc}
\lambda & 0 \\
0 & -\lambda
\end{array}\right)\right)=\left\{\left(\begin{array}{ll}
a & 0 \\
0 & b
\end{array}\right) \mid a, b \in \mathbb{F}_{q}, a b=1\right\}
$$

and matrices of type $2 \mathrm{a}$ are regular.

Type $2 \mathrm{~b}$ consists of semisimple matrices with eigenvalues $\lambda, \lambda^{q} \in \mathbb{F}_{q^{2}} \backslash \mathbb{F}_{q}$. The minimal polynomial of such elements over $\mathbb{F}_{q}$ is equal to $(X-\lambda)\left(X-\lambda^{q}\right)$. The centraliser of a typical element is isomorphic to the group of elements of norm 1 in the field $\mathbb{F}_{q^{2}}$ and matrices of type $2 \mathrm{~b}$ are regular.

\section{Appendix B. Adjoint ACtion OF $\mathrm{GL}_{3}\left(\mathbb{F}_{q}\right)$ ON $\mathfrak{s l}_{3}\left(\mathbb{F}_{q}\right)$}

This appendix is almost identical to Appendix B in [3] and included for the reader's convenience. Let $\mathbb{F}_{q}$ be a finite field of characteristic not equal to 3 . We give an overview of the elements in $\mathfrak{s l}_{3}\left(\mathbb{F}_{q}\right)$ up to conjugacy under the group $\mathrm{GL}_{3}\left(\mathbb{F}_{q}\right)$. For this we distinguish eight different types, labelled $0,1,2,3,4 \mathrm{a}, 4 \mathrm{~b}, 4 \mathrm{c}, 5$. The total number of elements of each type and the isomorphism types of their centralisers in $\mathrm{SL}_{3}\left(\mathbb{F}_{q}\right)$ are summarised in Tables 7.1 and 7.2 in [3]. We briefly discuss the eight different types.

Type 0 consists of the zero matrix, which does not feature in our calculation but is shown for completeness. Its centraliser is the entire group $\mathrm{SL}_{3}\left(\mathbb{F}_{q}\right)$.

Type 1 consists of nilpotent matrices with minimal polynomial equal to $X^{3}$ over $\mathbb{F}_{q}$. The centraliser of a typical element is

$$
\mathrm{C}_{\mathrm{SL}_{3}\left(\mathbb{F}_{q}\right)}\left(\left(\begin{array}{lll}
0 & 1 & 0 \\
0 & 0 & 1 \\
0 & 0 & 0
\end{array}\right)\right)=\left\{\left(\begin{array}{lll}
a & b & c \\
0 & a & b \\
0 & 0 & a
\end{array}\right) \in \mathrm{GL}_{3}\left(\mathbb{F}_{q}\right) \mid a^{3}=1\right\}
$$

and matrices of type 1 are regular.

Type 2 consists of nilpotent matrices with minimal polynomial equal to $X^{2}$ over $\mathbb{F}_{q}$. The centraliser of a typical element is

$$
\mathrm{C}_{\mathrm{SL}_{3}\left(\mathbb{F}_{q}\right)}\left(\left(\begin{array}{lll}
0 & 1 & 0 \\
0 & 0 & 0 \\
0 & 0 & 0
\end{array}\right)\right)=\left\{\left(\begin{array}{lll}
a & b & c \\
0 & a & 0 \\
0 & d & e
\end{array}\right) \in \mathrm{GL}_{3}\left(\mathbb{F}_{q}\right) \mid a^{2} e=1\right\}
$$

and matrices of type 2 are irregular.

Type 3 consists of semisimple matrices with eigenvalues $\lambda \in \mathbb{F}_{q} \backslash\{0\}$ of multiplicity 2 and $\mu:=-2 \lambda$. The minimal polynomial of such elements over $\mathbb{F}_{q}$ is equal to $(X-$ $\lambda)(X-\mu)$. The centraliser of a typical element is

$$
\mathrm{C}_{\mathrm{SL}_{3}\left(\mathbb{F}_{q}\right)}\left(\left(\begin{array}{ccc}
\lambda & 0 & 0 \\
0 & \lambda & 0 \\
0 & 0 & \mu
\end{array}\right)\right)=\left\{\left(\begin{array}{ccc}
a & b & 0 \\
c & 0 & 0 \\
0 & 0 & e
\end{array}\right) \in \mathrm{GL}_{3}\left(\mathbb{F}_{q}\right) \mid(a d-b c) e=1\right\}
$$

and matrices of type 3 are irregular.

Type $4 \mathrm{a}$ consists of semisimple matrices with distinct eigenvalues $\lambda, \mu, \nu:=-\lambda-\mu \in$ $\mathbb{F}_{q} \backslash\{0\}$. The minimal polynomial of such elements over $\mathbb{F}_{q}$ is equal to $(X-\lambda)(X-$ $\mu)(X-\nu)$. The centraliser of a typical element is

$$
\mathrm{C}_{\mathrm{SL}_{3}\left(\mathbb{F}_{q}\right)}\left(\left(\begin{array}{ccc}
\lambda & 0 & 0 \\
0 & \mu & 0 \\
0 & 0 & \nu
\end{array}\right)\right)=\left\{\left(\begin{array}{ccc}
a & 0 & 0 \\
0 & b & 0 \\
0 & 0 & c
\end{array}\right) \in \mathrm{GL}_{3}\left(\mathbb{F}_{q}\right) \mid a b c=1\right\}
$$


and matrices of type $4 \mathrm{a}$ are regular.

Type 4b consists of semisimple matrices with eigenvalues $\lambda, \mu:=\lambda^{q} \in \mathbb{F}_{q^{2}} \backslash \mathbb{F}_{q}$ and $\nu:=-\lambda-\mu \in \mathbb{F}_{q}$. The minimal polynomial of such elements over $\mathbb{F}_{q}$ is equal to $(X-\lambda)(X-\mu)(X-\nu)$. The centraliser of a typical element is isomorphic to the multiplicative group of the field $\mathbb{F}_{q^{2}}$ and matrices of type $4 \mathrm{~b}$ are regular.

Type 4c consists of semisimple matrices with eigenvalues $\lambda, \mu:=\lambda^{q}, \nu:=\lambda^{q^{2}} \in \mathbb{F}_{q^{3}} \backslash \mathbb{F}_{q}$ with $\lambda+\mu+\nu=0$. The minimal polynomial of such elements over $\mathbb{F}_{q}$ is equal to $(X-\lambda)(X-\mu)(X-\nu)$. The centraliser of a typical element is isomorphic to the group of elements of norm 1 in the field $\mathbb{F}_{q^{3}}$ and matrices of type $4 \mathrm{c}$ are regular.

Type 5 consists of matrices with eigenvalues $\lambda \in \mathbb{F}_{q} \backslash\{0\}$ of multiplicity 2 and $\mu:=$ $-2 \lambda$. The minimal polynomial of such elements over $\mathbb{F}_{q}$ is equal to $(X-\lambda)^{2}(X-\mu)$. The centraliser of a typical element is

$$
\mathrm{C}_{\mathrm{SL}_{3}\left(\mathbb{F}_{q}\right)}\left(\left(\begin{array}{lll}
\lambda & 1 & 0 \\
0 & \lambda & 0 \\
0 & 0 & \mu
\end{array}\right)\right)=\left\{\left(\begin{array}{ccc}
a & b & 0 \\
0 & a & 0 \\
0 & 0 & c
\end{array}\right) \in \mathrm{GL}_{3}\left(\mathbb{F}_{q}\right) \mid a^{2} c=1\right\}
$$

and matrices of type 5 are regular.

\section{Appendix C. Auxiliary Results Regarding $\mathfrak{s l}_{3}\left(\mathbb{F}_{q}\right)$ And $\mathfrak{g l}_{3}\left(\mathbb{F}_{q}\right)$ FOR $q \equiv_{3} 0$}

C.1. Let $\mathbb{F}_{q}$ be a finite field of characteristic equal to 3. In Section 5, we require an overview of the elements in $\mathfrak{s l}_{3}\left(\mathbb{F}_{q}\right)$ up to conjugacy under the group $\mathrm{GL}_{3}\left(\mathbb{F}_{q}\right)$. We distinguish six different types, labelled A, B, C, D, E and F, corresponding to the types $0,1,2,4 \mathrm{a}, 4 \mathrm{~b}$ and $4 \mathrm{c}$ in the generic case $(p \neq 3)$; cf. Appendix $\mathrm{B}$. There are no analogues to types 3 and 5 in characteristic 3 . The total number of elements of each type, number of orbits and orbit sizes, are summarised in Table 5.1. We briefly discuss the six different types.

Type A (corresponding to type 0 in the generic case) consists of the scalar matrices. This type is listed for completeness and does not feature in our calculation.

Type B (corresponding to type 1) consists of matrices with minimal polynomial equal to $(X-\lambda)^{3}$ over $\mathbb{F}_{q}$. There are $q$ possible values for $\lambda \in \mathbb{F}_{q}$, hence $q$ orbits. The orbit sizes are as in the generic case; see (B.1). In contrast to the generic case, matrices of type $\mathrm{B}$ are irregular in characteristic 3 .

Type $\mathrm{C}$ (corresponding to type 2 ) consists of matrices with minimal polynomial equal to $(X-\lambda)^{2}$ over $\mathbb{F}_{q}$. There are $q$ possible values for $\lambda \in \mathbb{F}_{q}$, hence $q$ orbits. The orbit sizes are as in the generic case; see (B.2). In contrast to the generic case, matrices of type $\mathrm{B}$ are irregular in characteristic 3 .

Type D (corresponding to type 4a) consists of matrices with minimal polynomial equal to $(X-\lambda)(X-\mu)(X-\nu)$ over $\mathbb{F}_{q}$, with distinct $\lambda, \mu, \nu$ such that $\lambda+\mu+\nu=0$. There are $(q-1) q / 6$ possible choices for $\{\lambda, \mu, \nu\}$, hence the same number of orbits. The orbit sizes are as in the generic case; see (B.4). As in the generic case, matrices of type $\mathrm{D}$ are regular.

Type $\mathrm{E}$ (corresponding to type $4 \mathrm{~b}$ ) consists of semisimple matrices with eigenvalues $\lambda, \mu:=\lambda^{q} \in \mathbb{F}_{q^{2}} \backslash \mathbb{F}_{q}$ and $\nu:=-\lambda-\mu \in \mathbb{F}_{q}$. The number of orbits and the orbit sizes are exactly as in the generic case; see Appendix B. Matrices of type E are regular.

Type $\mathrm{F}$ (corresponding to type 4c) consists of semisimple matrices with eigenvalues $\lambda, \mu:=\lambda^{q}, \nu:=\lambda^{q^{2}} \in \mathbb{F}_{q^{3}} \backslash \mathbb{F}_{q}$ with $\lambda+\mu+\nu=0$. In characteristic 3 , the number of elements in $\mathbb{F}_{q^{3}} \backslash \mathbb{F}_{q}$ with trace 0 in $\mathbb{F}_{q}$ is $q^{2}-q$. Thus there are $(q-1) q / 3$ orbits. The orbit sizes are as in the generic case; see Appendix B. Matrices of type $\mathrm{F}$ are regular. 
C.2. Let $\mathbb{F}_{q}$ be a finite field of characteristic equal to 3. In Section 5 , we also require an overview of the elements in $\left\{\mathbf{x}+\mathbb{F}_{q} \overline{\mathbf{z}} \mid \mathbf{x} \in \mathfrak{g l}_{3}\left(\mathbb{F}_{q}\right)\right.$ with $\left.\operatorname{Tr}(\mathbf{x})=-1\right\}$ up to conjugacy under the group $\mathrm{GL}_{3}\left(\mathbb{F}_{q}\right)$. We distinguish five different types, labelled $\mathrm{G}, \mathrm{H}, \mathrm{I}, \mathrm{J}$ and $\mathrm{K}$, corresponding to the types $3,4 \mathrm{a}, 4 \mathrm{~b}, 4 \mathrm{c}$ and 5 in the generic case $(p \neq 3)$; cf. Appendix $\mathrm{B}$. There are no analogues to types 1 and 2 in characteristic 3 . The total number of elements of each type, number of orbits and orbit sizes, are summarised in Table 5.2. We briefly discuss the five different types.

Type $\mathrm{G}$ (analogous to type 3 in the generic case) consists of semisimple matrices with eigenvalues $\lambda \in \mathbb{F}_{q}$ of multiplicity 2 and $\mu:=\lambda-1$. The minimal polynomial of such an element over $\mathbb{F}_{q}$ is equal to $(X-\lambda)(X-\mu)$. There are $q$ possible values for $\lambda$, hence $q$ orbits. The orbit sizes are as in the generic case; see (B.3). As in the generic case, matrices of type $\mathrm{G}$ are irregular.

Type $\mathrm{H}$ (analogous to type 4a) consists of matrices with minimal polynomial equal to $(X-\lambda)(X-\mu)(X-\nu)$ over $\mathbb{F}_{q}$, with distinct $\lambda, \mu, \nu$ such that $\lambda+\mu+\nu=-1$. One checks that there are $(q-3) q / 6$ possible choices for $\{\lambda, \mu, \nu\}$, hence the same number of orbits. The orbit sizes are as in the generic case; see (B.4). As in the generic case, matrices of type $\mathrm{H}$ are regular.

Type I (analogous to type $4 \mathrm{~b}$ ) consists of semisimple matrices with eigenvalues $\lambda, \mu:=$ $\lambda^{q} \in \mathbb{F}_{q^{2}} \backslash \mathbb{F}_{q}$ and $\nu:=-\lambda-\mu-1 \in \mathbb{F}_{q}$. The number of orbits and the orbit sizes are exactly as in the generic case; see Appendix B. Matrices of type I are regular.

Type $\mathrm{J}$ (analogous to type 4c) consists of semisimple matrices with eigenvalues $\lambda, \mu:=$ $\lambda^{q}, \nu:=\lambda^{q^{2}} \in \mathbb{F}_{q^{3}} \backslash \mathbb{F}_{q}$ with $\lambda+\mu+\nu=-1$. In characteristic 3 , the number of elements in $\mathbb{F}_{q^{3}} \backslash \mathbb{F}_{q}$ with trace -1 in $\mathbb{F}_{q}$ is $q^{2}$. Thus there are $q^{2} / 3$ orbits. The orbit sizes are as in the generic case; see Appendix B. Matrices of type $\mathrm{J}$ are regular.

Type $\mathrm{K}$ (analogous to type 5 ) consists of matrices with eigenvalues $\lambda \in \mathbb{F}_{q}$ of multiplicity 2 and $\mu:=\lambda-1$. The minimal polynomial of such an element over $\mathbb{F}_{q}$ is equal to $(X-\lambda)^{2}(X-\mu)$. There are $q$ possible values for $\lambda$, hence $q$ orbits. The orbit sizes are as in the generic case; see (B.5). As in the generic case, matrices of type $\mathrm{K}$ are irregular.

Acknowledgements. The authors would like to thank Alexander Lubotzky as well as the following institutions: the Batsheva de Rothschild Fund for the Advancement of Science, the EPSRC, the Mathematisches Forschungsinstitut Oberwolfach, the National Science Foundation and the Nuffield Foundation.

\section{REFERENCES}

[1] C. A. M. André, A. P. Nicolás, Supercharacters of the adjoint group of a finite radical ring, J. Group Theory 11 (2008), 709-746.

[2] N. Avni, B. Klopsch, U. Onn, C. Voll, On representation zeta functions of groups and a conjecture of Larsen-Lubotzky, C. R. Math. Acad. Sci. Paris 348 (2010), 363-367.

[3] N. Avni, B. Klopsch, U. Onn, C. Voll, Representation zeta functions of compact p-adic analytic groups and arithmetic groups, arXiv:1007.2900v1 (2010).

[4] N. Avni, B. Klopsch, U. Onn, C. Voll, Representation zeta functions for $\mathrm{SL}_{3}$, preprint.

[5] L. Bartholdi, P. de la Harpe, Representation zeta functions of wreath products with finite groups, Groups Geom. Dyn. 4 (2010), 209-249.

[6] W. Borho, H. Kraft, Über Bahnen und deren Deformationen bei linearen Aktionen reduktiver Gruppen, Comment. Math. Helvetici 54 (1979), 61-104.

[7] J. Denef, Report on Igusa's local zeta function, Séminaire Bourbaki, Vol. 1990/91. 
[8] J. González-Sánchez, Kirillov's orbit method for p-groups and pro-p groups, Comm. Algebra 37 (2009), 4476-4488.

[9] E. Hrushovski, B. Martin, Zeta functions from definable equivalence relations, arXiv:math/070111 (2007).

[10] B. Huppert, Character theory of finite groups, de Gruyter Expositions in Mathematics 25, Walter de Gruyter \& Co., Berlin, 1998.

[11] J. Igusa, An introduction to the theory of local zeta functions, AMS/IP Studies in Advanced Mathematics 14, American Mathematical Society, Providence, RI, International Press, Cambridge, MA, 2000.

[12] A. Jaikin-Zapirain, Zeta functions of representations of compact p-adic analytic groups, J. Amer. Math. Soc. 19 (2006), 91-118.

[13] G. Klaas, C. R. Leedham-Green, W. Plesken, Linear pro-p-groups of finite width, Lecture Notes in Mathematics 1674, Springer-Verlag, Berlin, 1997.

[14] B. Klopsch, On the Lie theory of p-adic analytic groups, Math. Z. 249 (2005), 713-730.

[15] M. Lazard, Groupes analytiques p-adiques, Publ. Math. IHÉS 26 (1965), 389-603.

[16] M. Larsen, A. Lubotzky, Representation growth of linear groups, J. Eur. Math. Soc. (JEMS) 10 (2008), 351-390.

[17] A. Nobs, J. Wolfart, Die irreduziblen Darstellungen der Gruppen $\mathrm{SL}_{2}\left(\mathbb{Z}_{p}\right)$, insbesondere $\mathrm{SL}_{2}\left(\mathbb{Z}_{2}\right)$. II., Comment. Math. Helv. 51 (1976), 491-526.

[18] G. Prasad, M. S. Raghunathan, Topological central extensions of $\mathrm{SL}_{1}(D)$, Invent. Math. 92 (1988), 645-689.

[19] C. Voll, Functional equations for zeta functions of groups and rings, Ann. of Math. (2) 172 (2010), 1181-1218.

[20] W. Wang, Dimension of a minimal nilpotent orbit, Proc. Amer. Math. Soc. 127 (1999), 935-936.

Department of Mathematics, Harvard University, One Oxford Street, Cambridge MA 02138, USA

E-mail address: avni.nir@gmail.com

Department of Mathematics, Royal Holloway, University of London, Egham TW20 0EX, United KINGDOM

E-mail address: Benjamin.Klopsch@rhul.ac.uk

Department of Mathematics, Ben Gurion University of the Negev, Beer-Sheva 84105 ISRAEL

E-mail address: urionn@math.bgu.ac.il

School of Mathematics, University of Southampton, University Road, Southampton SO17 1BJ, United Kingdom

E-mail address: C.Voll.98@cantab.net 